\title{
Sard property for the endpoint map on some Carnot groups
}

\author{
Enrico Le Donne ${ }^{\mathrm{a}, *}$, Richard Montgomery ${ }^{\mathrm{b}}$, Alessandro Ottazzi $^{\mathrm{c}, \mathrm{d}}$, Pierre Pansu $^{\mathrm{e}}$, \\ Davide Vittone ${ }^{\mathrm{f}, \mathrm{g}}$ \\ a Department of Mathematics and Statistics, University of Jyväskylä, Jyväskylä, Finland \\ ${ }^{\mathrm{b}}$ Mathematics Department, University of California, Santa Cruz, USA \\ ${ }^{\mathrm{c}}$ Dipartimento di Matematica, Università di Trento, Trento, Italy \\ ${ }^{\mathrm{d}}$ School of Mathematics and Statistics, University of New South Wales, Sydney, Australia \\ e Département de Mathématiques, Université Paris-Sud, Orsay, France \\ ${ }^{\mathrm{f}}$ Dipartimento di Matematica Pura ed Applicata, Università di Padova, Padova, Italy \\ g Institut für Mathematik, Universität Zürich, Zürich, Switzerland
}

Received 13 March 2015; received in revised form 10 July 2015; accepted 30 July 2015

Available online 28 August 2015

\begin{abstract}
In Carnot-Carathéodory or sub-Riemannian geometry, one of the major open problems is whether the conclusions of Sard's theorem holds for the endpoint map, a canonical map from an infinite-dimensional path space to the underlying finite-dimensional manifold. The set of critical values for the endpoint map is also known as abnormal set, being the set of endpoints of abnormal extremals leaving the base point. We prove that a strong version of Sard's property holds for all step-2 Carnot groups and several other classes of Lie groups endowed with left-invariant distributions. Namely, we prove that the abnormal set lies in a proper analytic subvariety. In doing so we examine several characterizations of the abnormal set in the case of Lie groups.

(c) 2015 L'Association Publications de l'Institut Henri Poincaré. Published by Elsevier B.V. All rights reserved.
\end{abstract}

MSC: 53C17; 22F50; 22E25; 14M17

Keywords: Sard's property; Endpoint map; Abnormal curves; Carnot groups; Polarized groups; Sub-Riemannian geometry

\section{Introduction}

Let $G$ be a connected Lie group with Lie algebra $\mathfrak{g}$. Let $V \subseteq \mathfrak{g}$ be a subspace. Following Gromov [12, Sec. 0.1], we shall call the pair $(G, V)$ a polarized group. Carnot groups are examples of polarized groups where $V$ is the first layer of their stratification. To any polarized group $(G, V)$ one associates the endpoint map:

$$
\text { End : } \begin{aligned}
L^{2}([0,1], V) & \rightarrow G \\
u & \mapsto \gamma_{u}(1),
\end{aligned}
$$

\footnotetext{
* Corresponding author.

E-mail addresses: enrico.ledonne@jyu.fi (E. Le Donne), rmont@ucsc.edu (R. Montgomery), alessandro.ottazzi@gmail.com (A. Ottazzi), Pierre.Pansu@math.u-psud.fr (P. Pansu), vittone@math.unipd.it (D. Vittone).
} 
where $\gamma_{u}$ is the curve on $G$ leaving from the origin $e \in G$ with derivative $\left(\mathrm{d} L_{\gamma_{u}(t)}\right)_{e} u(t)$, where $L_{g}$ denotes the left translation by $g$.

The abnormal set of $(G, V)$ is the subset $\operatorname{Abn}(e) \subset G$ of all singular values of the endpoint map. Equivalently, $\operatorname{Abn}(e)$ is the union of all abnormal curves passing through the origin (see Section 2.3). If the abnormal set has measure 0 , then $(G, V)$ is said to satisfy the Sard Property. Proving the Sard Property in the general context of polarized manifolds is one of the major open problems in sub-Riemannian geometry, see the questions in [17, Sec. 10.2] and Problem III in [4]. In this paper, we will focus on the following stronger versions of Sard's property in the context of groups.

Definition 1.1 (Algebraic and Analytic Sard Property). We say that a polarized group $(G, V)$ satisfies the Algebraic (respectively, Analytic) Sard Property if its abnormal set $\operatorname{Abn}(e)$ is contained in a proper real algebraic (respectively, analytic) subvariety of $G$.

Our main results are summarized by:

Theorem 1.2. The following Carnot groups satisfy the Algebraic Sard Property:

(1) Carnot groups of step 2;

(2) The free-nilpotent group of rank 3 and step 3;

(3) The free-nilpotent group of rank 2 and step 4;

(4) The nilpotent part of the Iwasawa decomposition of any semisimple Lie group equipped with the distribution defined by the sum of the simple root spaces.

The following polarized groups satisfy the Analytic Sard Property:

(5) Split semisimple Lie groups equipped with the distribution given by the subspace of the Cartan decomposition with negative eigenvalue.

(6) Split semisimple Lie groups equipped with the distribution defined by the sum of the nonzero root spaces.

Earlier work [16] allows us

(7) compact semisimple Lie groups equipped with the distribution defined by the sum of the nonzero root spaces, (i.e., the orthogonal to the maximal torus relative to a bi-invariant metric).

Case (1) will be proved reducing the problem to the case of a smooth map between finite-dimensional manifolds and applying the classical Sard Theorem to this map. The proof will crucially use the fact that in a Carnot group of step 2 each abnormal curve is contained in a proper subgroup. This latter property may fail for step 3, see Section 6.3. However, a similar strategy together with the notion of abnormal varieties, see (2.20), might yield a proof of Sard Property for general Carnot groups.

The proof of cases (2)-(6) is based on the observation that, if $\mathcal{X}$ is a family of contact vector fields (meaning infinitesimal symmetries of the distribution) vanishing at the identity, then for any horizontal curve $\gamma$ leaving from the origin with control $u$ we have

$$
\left(R_{\gamma(1)}\right)_{*} V+\left(L_{\gamma(1)}\right)_{*} V+\mathcal{X}(\gamma(1)) \subset \operatorname{Im}\left(\operatorname{dEnd}_{u}\right) \subset T_{\gamma(1)} G .
$$

Therefore if $g \in G$ is such that

$$
\left(R_{g}\right)_{*} V+\left(L_{g}\right)_{*} V+\mathcal{X}(g)=T_{g} G,
$$

then $g$ is not a singular value of the endpoint map. In fact, if (1.3) is describable as a non-trivial system of polynomial inequations for $g$, then $(G, V)$ has the Algebraic Sard Property. Case (3) was already proved in [15] by using an equivalent technique. 
Equation (1.3) does not have solutions in the following cases: free-nilpotent groups of rank 2 and step $\geq 5$, freenilpotent groups of rank 3 and step $\geq 4$, free-nilpotent groups of rank $\geq 4$ and step $\geq 3$. Here Sard's property remains an open problem.

We further provide a more quantitative version of Sard's property for free-nilpotent groups of step 2.

Theorem 1.4. In any free-nilpotent group of step 2 the abnormal set is contained in an affine algebraic subvariety of codimension 3.

Agrachev, Gentile and Lerario proved that in a generic Carnot group of step 2 the generic point in the second layer is not in the abnormal set, see [2, Theorem 9].

There are several papers that give a bound on the size of the set of all those points $\operatorname{End}(u)$ where $u$ is a critical point with the extra property that $\gamma_{u}$ is length minimizing for a fixed sub-Riemannian structure. A very general result [3] by Agrachev based on techniques of Rifford and Trélat [18] states that this set is contained in a closed nowhere dense set, for general sub-Riemannian manifolds.

In this direction, in step 3 Carnot groups equipped with a sub-Riemannian structure on the first layer, we bound the size of the set $\mathrm{Abn}^{l m}(e)$ of points connected to the origin by locally length minimizing abnormal curves. Our result uses ideas of Tan and Yang [19] and the fact that in an arbitrary polarized Lie group the Sard Property holds for normal-abnormal curves, see Lemma 2.31.

Theorem 1.5. Let $G$ be a sub-Riemannian Carnot group of step 3. The sub-analytic Sard Property holds for locally length minimizing abnormal curves. Namely, the set $\mathrm{Abn}^{l m}(e)$ is contained in a sub-analytic set of codimension at least 1 .

The paper is organized as follows. Section 2 is a preliminary section. First we recall the definition of the endpoint map and we give a characterization of the image of its differential in Proposition 2.3, in the case of polarized groups. Secondly, we review Carnot groups, abnormal curves, and give interpretations of the abnormal equations using leftinvariant forms and right-invariant forms. In Section 2.5, we examine the notion of abnormal varieties. In Section 2.7 we review normal curves, and in Section 2.8 we review the Goh condition. In Section 3 we consider step-2 Carnot groups. We first prove the Algebraic Sard Property for general Carnot groups of step 2 and then we prove Theorem 1.4 for free step- 2 groups. For the latter, we also give precise characterizations of the abnormal set. In Section 4 we discuss sufficient conditions for Sard's property to hold. In particular, we discuss the role of contact vector fields and equation (1.3). The most important criteria are Proposition 4.11 and Corollary 4.14, which will be used in Section 5 to prove the remaining part of Theorem 1.2. In Section 5.3 we discuss Sard Property for a large class of semidirect products of polarized groups. In particular, we provide examples of groups with exponential growth having the Analytic Sard Property (semisimple Lie groups) and the Algebraic Sard Property (solvable Lie groups). See Proposition 5.5 and Remark 5.6. Section 6 is devoted to Carnot groups of step 3. First we prove Sard Property for abnormal length minimizers, i.e., Theorem 1.5. Second, we investigate the example of the free 3-step rank-3 Carnot group, showing that the argument used in step-2 Carnot groups finds an obstruction: there are abnormal curves not contained in any proper subgroup. We conclude the article with Section 7, where we discuss the open problems.

\section{Preliminaries}

Let $G$ be a connected Lie group with Lie algebra $\mathfrak{g}$, viewed as the tangent space of $G$ at the identity element $e$. For all $g \in G$, denote by $L_{g}$ and $R_{g}$ the left and right multiplication by $g$, respectively. Also, $\operatorname{Ad}_{g}:=\mathrm{d}\left(L_{g} \circ R_{g^{-1}}\right)_{e}$.

Fix a linear subspace $V \subseteq \mathfrak{g}$. Let $u$ be an element of $L^{2}([0,1], V)$. Denote by $\gamma_{u}$ the curve in $G$ that solves the ODE:

$$
\frac{\mathrm{d} \gamma}{\mathrm{d} t}(t)=\left(\mathrm{d} L_{\gamma(t)}\right)_{e} u(t)
$$

with initial condition $\gamma(0)=e$. Viceversa, if $\gamma:[0,1] \rightarrow G$ is an absolutely continuous curve that solves (2.1) for some $u \in L^{2}([0,1], V)$, then we say that $\gamma$ is horizontal with respect to $V$ and that $u=u_{\gamma}$ is its control. In other words, the derivatives of $\gamma$ lie in the left-invariant subbundle, denoted by $\Delta$, that coincides with $V$ at $e$. 
The endpoint map starting at $e$ with controls in $V$ is the map

$$
\text { End : } \begin{aligned}
L^{2}([0,1], V) & \rightarrow G \\
u & \mapsto \gamma_{u}(1) .
\end{aligned}
$$

\subsection{Differential of the endpoint map}

The following result is standard and a proof of it can be found (in the more general context of Carnot-Carathéodory manifolds) in [17, Proposition 5.2.5, see also Appendix E].

Theorem 2.2 (Differential of End). The endpoint map End is a smooth map between the Hilbert space $L^{2}([0,1], V)$ and $G$. If $\gamma$ is a horizontal curve leaving from the origin with control $u$, then the differential of End at $u$, which is a map from $L^{2}([0,1], V)$ to the tangent space of $G$ at $\gamma(1)$, is given by

$$
\mathrm{dEnd}_{u} v=\left(\mathrm{d} R_{\gamma(1)}\right)_{e} \int_{0}^{1} \operatorname{Ad}_{\gamma(t)} v(t) \mathrm{d} t, \quad \forall v \in L^{2}([0,1], V) .
$$

Sketch of the proof. We sketch the proof for $G \subset G L_{n}(\mathbb{R})$, where we can interpret the Lie product as a matrix product and work in the matrix coordinates. Let $\gamma_{u+\epsilon v}$ be the curve with the control $u+\epsilon v$ and $\sigma(t)$ be the derivative of $\gamma_{u+\epsilon v}(t)$ with respect to $\epsilon$ at $\epsilon=0$. Then $\sigma$ satisfies the following ODE (which is the derivation with respect to $\epsilon$ of (2.1) for $\left.\gamma_{u+\epsilon v}\right)$

$$
\frac{\mathrm{d} \sigma}{\mathrm{d} t}=\gamma(t) \cdot v(t)+\sigma \cdot u(t)
$$

Now it is easy to see that $\int_{0}^{t} \operatorname{Ad}_{\gamma(s)}(v(s)) \mathrm{d} s \cdot \gamma(t)$ satisfies the above equation with the same initial condition as $\sigma$, hence is equal to $\sigma$.

Proposition 2.3 (Image of $\mathrm{d}$ End). If $\gamma:[0,1] \rightarrow G$ is a horizontal curve leaving from the origin with control $u$, then

$$
\operatorname{Im}\left(\mathrm{dEnd}_{u}\right)=\left(\mathrm{d} R_{\gamma(1)}\right)_{e}\left(\operatorname{span}\left\{\operatorname{Ad}_{\gamma(t)} V: t \in[0,1]\right\}\right) .
$$

Proof. A glance at the formula of Theorem 2.2 combined with the fact that $\left(\mathrm{d} R_{\gamma(1)}\right)_{e}$ is a linear isomorphism from $\mathfrak{g}$ to $T_{\gamma(1)} G$ shows that it suffices to prove that

$$
\left\{\int_{0}^{1} \operatorname{Ad}_{\gamma(t)} v(t) \mathrm{d} t: v \in L^{2}([0,1], V)\right\}=\operatorname{span}\left\{\operatorname{Ad}_{\gamma(t)} V: t \in[0,1]\right\} .
$$

$\subset$ : Any linear combination of terms $\operatorname{Ad}_{\gamma\left(t_{i}\right)} v_{i}$ is in the right hand set. Now an integral is a limit of finite sums and the right hand side is closed. Hence the right hand side contains the left hand side.

$\supset$ : It suffices to show that any element of the form $\xi=\operatorname{Ad}_{\gamma\left(t_{1}\right)} v_{1}$ lies in the left hand side. Let $\psi_{n}(t)$ be a deltafunction family centered at $t_{1}$, that is, a smooth family of continuous functions for which the limit as a distribution as $n \rightarrow \infty$ of $\psi_{n}(t)$ is $\delta\left(t-t_{1}\right)$. Then $\lim _{n \rightarrow \infty} \int_{0}^{1} \operatorname{Ad}_{\gamma(t)} \psi_{n}(t) v_{1} \mathrm{~d} t=\operatorname{Ad}_{\gamma\left(t_{1}\right)} v_{1}=\xi$ and since the left hand side is a closed subspace, $\xi$ lies in the set in the left hand side.

Remark 2.5. Evaluating (2.4) at $t=0$ and $t=1$ yields

$$
\left(\mathrm{d} R_{\gamma(1)}\right)_{e} V+\left(\mathrm{d} L_{\gamma(1)}\right)_{e} V \subset \operatorname{Im}\left(\mathrm{dEnd}_{u}\right) .
$$

Remark 2.7. Proposition 2.3 implies immediately that for strongly bracket generating distributions, the endpoint map is a submersion at every $u \neq 0$. We recall that a polarized group $(G, V)$ is strongly bracket generating if for every $X \in V \backslash\{0\}$, one has $V+[X, V]=\mathfrak{g}$. 
Remark 2.8 (Goh's condition is automatic in rank 2). Assume that $\operatorname{dim} V=2$. We claim that if $\gamma$ is horizontal leaving from the origin with control $u$, then for all $t \in[0,1]$ we have

$$
\left(\mathrm{d} R_{\gamma(1)}\right)_{e} \operatorname{Ad}_{\gamma(t)}[V, V] \subseteq \operatorname{Im}\left(\mathrm{dEnd}_{u}\right) .
$$

Indeed, we may assume that $\gamma$ is parametrized by arc length and that $t$ is a point of differentiability. Hence, $\gamma(t)^{-1} \gamma(t+\epsilon)=\exp (u(t) \epsilon+o(\epsilon))$. Notice that since $u(t) \in V \backslash\{0\}$ and $\operatorname{dim} V=2$, it follows that $[u(t), V]=[V, V]$.

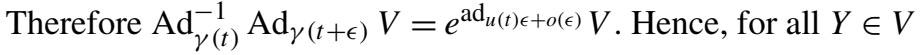

$$
\epsilon[u(t), Y]+o(\epsilon) \in V+\operatorname{Ad}_{\gamma(t)}^{-1} \operatorname{Ad}_{\gamma(t+\epsilon)} V .
$$

Therefore, Proposition 2.3 implies that $\operatorname{Ad}_{\gamma(t)}[u(t), Y] \in\left(\mathrm{d} R_{\gamma(1)}\right)_{e}^{-1} \operatorname{Im}\left(\mathrm{dEnd}_{u}\right)$, which proves the claim.

By (2.34) below, formula (2.9) implies that, whenever $\gamma$ is an abnormal curve (see Section 2.3) in a polarized group $(G, V)$ of rank 2, then $\gamma$ satisfies the Goh condition (see Section 2.8).

Remark 2.10 (Action of contact maps). We associate to the subspace $V \subseteq \mathfrak{g}$ a left-invariant subbundle $\Delta$ of $T G$ such that $\Delta_{e}=V$. A vector field $\xi \in \operatorname{Vec}(G)$ is said to be contact if its flow $\Phi_{\xi}^{s}$ preserves $\Delta$. Denote by

$$
\mathcal{S}:=\left\{\xi \in \operatorname{Vec}(G) \mid \xi \text { contact, } \xi_{e}=0\right\}
$$

the space of global contact vector fields on $G$ that vanish at the identity. We claim that, for every horizontal curve $\gamma$ leaving from the origin,

$$
\mathcal{S}(\gamma(1)) \subset \operatorname{Im}\left(\operatorname{dEnd}_{u}\right) .
$$

Indeed, let $\xi \in \mathcal{S}$ and let $\Phi_{\xi}^{s}$ be the corresponding flow at time $s$. Since $\xi_{e}=0$, we have that $\Phi_{\xi}^{s}(e)=e$. Consider the curve $\gamma^{s}:=\Phi_{\xi}^{s} \circ \gamma$. Notice that $\gamma^{s}(0)=e$ and that $\gamma^{s}$ is horizontal, because $\xi$ is a contact vector field. Therefore,

$$
\operatorname{End}\left(u^{s}\right)=\gamma^{s}(1)=\Phi_{\xi}^{s}(\gamma(1))
$$

where $u^{s}$ is the control of $\gamma^{s}$. Differentiating at $s=0$, we conclude that $\xi(\gamma(1))$, which is an arbitrary point in $\mathcal{S}(\gamma(1))$, belongs to $\operatorname{Im}\left(\mathrm{dEnd}_{u}\right)$.

\subsection{Carnot groups}

Among the polarized groups, Carnot groups are the most distinguished. A Carnot group is a simply connected, polarized Lie group $(G, V)$ whose Lie algebra $\mathfrak{g}$ admits a direct sum decomposition in nontrivial vector subspaces

$$
\mathfrak{g}=V_{1} \oplus V_{2} \oplus \ldots \oplus V_{s} \quad \text { such that } \quad\left[V_{i}, V_{j}\right]=V_{i+j}
$$

where $V_{k}=\{0\}, k>s$ and $V_{1}=V$. We refer to the $i$ th summand $V_{i}$ as the $i$ th layer.

The above decomposition is also called the stratification of $\mathfrak{g}$ and Carnot groups are often referred to in the analysis literature as stratified groups. The step of a Carnot group is the total number $s$ of layers and equals the degree of nilpotency of $\mathfrak{g}$ : all Lie brackets of length greater than $s$ vanish. Every Carnot group admits at least a canonical outer automorphism, the 'scaling' $\delta_{\lambda}$ which on $\mathfrak{g}$ is equal to the multiplication by $\lambda^{i}$ on the $i$ th layer.

Since $G$ is simply connected and nilpotent, the exponential map exp $: \mathfrak{g} \rightarrow G$ is a diffeomorphism. We write $\log$ for the inverse of exp. When we use $\log$ to identify $\mathfrak{g}$ with $G$ the group law on $G$ becomes a polynomial map $\mathfrak{g} \times \mathfrak{g} \rightarrow \mathfrak{g}$ with $0 \in \mathfrak{g}$ playing the role of the identity element $e \in G$.

\subsection{Abnormal curves}

Definition 2.12 (Abnormal curve). Let $(G, V)$ be a polarized group. Let $\gamma:[0,1] \rightarrow G$ be a horizontal curve leaving from the origin with control $u$. If $\operatorname{Im}\left(\mathrm{dEnd}_{u}\right) \subsetneq T_{\gamma(1)} G$, we say that $\gamma$ is abnormal.

In other words, $\gamma$ is abnormal if and only if $\gamma(1)$ is a critical value of End. We define the abnormal set of $(G, V)$ as 
$\operatorname{Abn}(e):=\{\gamma(1) \mid \gamma$ abnormal,$\gamma(0)=e\}=\{$ critical values of End $\}$.

The Sard Problem in sub-Riemannian geometry is the study of the above abnormal set. More information can be found in [17, page 182].

Interpretation of abnormal equations via right-invariant forms. Proposition 2.3 immediately gives an interpretation for a curve to be abnormal, which, to the best of our knowledge, is not in the literature.

Corollary 2.14. Let $(G, V)$ be a polarized group and let $\gamma:[0,1] \rightarrow G$ be a horizontal curve. Then the following are equivalent:

(1) $\gamma$ is abnormal;

(2) there exists $\lambda \in \mathfrak{g}^{*} \backslash\{0\}$ such that $\lambda\left(\operatorname{Ad}_{\gamma(t)} V\right)=\{0\}$ for every $t \in[0,1]$;

(3) there exists a right-invariant 1 -form $\alpha$ on $G$ such that $\alpha\left(\Delta_{\gamma(t)}\right)=\{0\}$ for every $t \in[0,1]$, where $\Delta$ is the leftinvariant distribution induced by $V$.

Interpretation of abnormal equations via left-invariant adjoint equations. The previous section characterized singular curves for a left-invariant distribution on a Lie group $G$ in terms of right-invariant one-forms. This section characterizes the same curves in terms of left-invariant one-forms. This left-invariant characterization is the one used in [16, Equations (12), (13) and (14)] and [11, equations in Section 2.3]. We establish the equivalence of the two characterizations directly using Lie theory. Then we take a second, Hamiltonian, perspective on the equivalence of characterizations. In this perspective, the right-invariant characterization is simply the momentum map applied to the Hamiltonian provided by the Maximum Principle.

We shall also introduce the notation

$$
w(\eta)(X, Y):=\eta([X, Y]), \text { for } \eta \in V^{\perp} \subset \mathfrak{g}^{*}, X, Y \in V .
$$

Proposition 2.16. Let $(G, V)$ be a polarized group and let $\gamma:[0,1] \rightarrow G$ be a horizontal curve with control $u$. Then the following are equivalent:

(1) $\gamma$ is abnormal;

(2) there exists a curve $\eta:[0,1] \rightarrow \mathfrak{g}^{*}$, with $\left.\eta(t)\right|_{V}=0$ and $\eta(t) \neq 0$, for all $t \in[0,1]$, representing a curve of left-invariant one-forms, such that

$$
\left\{\begin{array}{l}
\frac{\mathrm{d} \eta}{\mathrm{d} t}(t)=\left(\operatorname{ad}_{u(t)}\right)^{*} \eta(t) \\
u(t) \in \operatorname{Ker}(w(\eta(t)))
\end{array}\right.
$$

We remark that the first equation of (2) above has a sign difference with respect to the corresponding formula in [16, Sec. 4], the reason being that in the latter one considers the differential of the co-adjoint action $g \mapsto \operatorname{Ad}_{g^{-1}}$.

Golé and Karidi made good use of the coordinate version of the previous proposition. See [11, p. 540], following [16, Sec. 4]. See also $[14,15]$. To describe their version, fix a basis $X_{1}, \ldots, X_{n}$ of $\mathfrak{g}$ such that $X_{1}, \ldots, X_{r}$ is a basis of $V$. Let $c_{i j}^{k}$ be the structure constant of $\mathfrak{g}$ with respect to this basis, seen as left-invariant vector fields. Let $\left(u_{1}, \ldots, u_{r}\right) \in V$ be controls relative to this basis. Let $\eta_{i}=\eta\left(X_{i}\right)$ denote the linear coordinates of a covector $\eta \in \mathfrak{g}^{*}$ relative to this basis.

Proposition 2.17. Let $(G, V)$ be a polarized group. Let $\gamma:[0,1] \rightarrow G$ be a horizontal curve with control $\sum_{i=1}^{r} u_{i}(t) X_{i}$. Under the above coordinate conventions, the following are equivalent:

(1) $\gamma$ is abnormal;

(2) there exists a vector function $\left(0,0, \ldots, 0, \eta_{r+1}, \ldots, \eta_{n}\right):[0,1] \rightarrow \mathbb{R}^{n}$, never vanishing, such that

$$
\begin{cases}\frac{\mathrm{d} \eta_{i}}{\mathrm{~d} t}(t)+\sum_{j=1}^{r} \sum_{k=r+1}^{n} c_{i j}^{k} u_{j}(t) \eta_{k}(t)=0, & \text { for all } i=r+1, \ldots, n, \\ \sum_{j=1}^{r} \sum_{k=r+1}^{n} c_{i j}^{k} u_{j}(t) \eta_{k}(t)=0, & \text { for all } i=1, \ldots, r .\end{cases}
$$


Both Corollary 2.14 and Proposition 2.16 lead to a one-form $\lambda(t) \in T_{\gamma(t)}^{*} G$ along the curve $\gamma$ in $G$. The key to the equivalence of the right and left perspectives of these two propositions is that these one-forms along $\gamma$ are equal. For the right-invariant version, Corollary 2.14 provides first the constant covector $\lambda^{R} \in \mathfrak{g}^{*}=T_{e}^{*} G$, and then its right-invariant extension. Finally we evaluate this extension along $\gamma$. For the left-invariant version, following Proposition 2.16, we take the curve of covectors $\eta(t)$, consider their left-invariant extensions, say $\eta(t)^{L}$ (leading to a curve of left-invariant one-forms) and finally we evaluate $\eta(t)^{L}$ at $\gamma(t)$. The following lemma establishes that the forms obtained in these two different ways coincide along $\gamma$.

Lemma 2.18. Let $\gamma(t)$ be the curve in $G$ starting at e and having control $u(t)$. Let $\lambda(t)$ be a one-form defined along $\gamma$. Let $\lambda^{R}(t)=\left(R_{\gamma(t)}\right)^{*} \lambda(t) \in \mathfrak{g}^{*}$ be this one-form viewed by right-trivializing $T^{*} G$. Let $\eta(t)=\left(L_{\gamma(t)}\right)^{*} \lambda(t) \in \mathfrak{g}^{*}$ be this same one-form viewed by left-trivializing $T^{*} G$. Then $\lambda^{R}(t)$ is constant if and only if $\eta(t)$ solves the time-dependent linear differential equation $d \eta / d t=\left(\operatorname{ad}_{u(t)}\right)^{*} \eta(t)$ with initial condition $\eta(0)=\lambda(0)$.

Proof. Suppose that $\lambda^{R}(t)$ is constant: $\lambda^{R}(t) \equiv \lambda^{R}$. Set $g=\gamma(t)$. Then $\lambda(t)=\left(R_{g}^{-1}\right)^{*} \lambda^{R}$ and consequently $\eta(t)=$ $\left(L_{g}\right)^{*}\left(R_{g}^{-1}\right)^{*} \lambda^{R}=\left(\operatorname{Ad}_{g}\right)^{*} \lambda^{R}$. For small $\Delta t$ we write $\gamma(t+\Delta t)=\gamma(t)\left(\gamma(t)^{-1} \gamma(t+\Delta t)\right)=g h$ with $h=h(\Delta t)=$ $\gamma(t)^{-1} \gamma(t+\Delta t)$ and use $\left(\operatorname{Ad}_{g h}\right)^{*}=\left(\operatorname{Ad}_{h}\right)^{*}\left(\operatorname{Ad}_{g}\right)^{*}$ to establish the identity for the difference quotient:

$$
\frac{1}{\Delta t}(\eta(t+\Delta t)-\eta(t))=\frac{1}{\Delta t}\left(\left(\operatorname{Ad}_{h(\Delta t)}\right)^{*}-\mathrm{Id}\right) \eta(t) .
$$

Now we use that the derivative of the adjoint representation $h \mapsto \operatorname{Ad}_{h}$ evaluated at the identity, is the standard adjoint representation $\mathfrak{g} \rightarrow \mathfrak{g l}(\mathfrak{g}), X \rightarrow \operatorname{ad}_{X}=[X, \cdot]$. Taking duals, we see that the difference quotient $\frac{1}{\Delta t}\left(\left(\operatorname{Ad}_{h(\Delta t)}\right)^{*}-\mathrm{Id}\right)$ limits to the linear operator $\left(\operatorname{ad}_{u(t)}\right)^{*}$ on $\mathfrak{g}^{*}$.

The steps just taken are reversed with little pain, showing the equivalence.

\subsection{Hamiltonian formalism and reduction}

We describe the Hamiltonian perspective on Corollary 2.14, Proposition 2.16 and the relation between them.

We continue with the basis $X_{i}$ of left-invariant vector fields on $G$, labelled so that the first $r$ form a basis of $V$. Write $P_{i}: T^{*} G \rightarrow \mathbb{R}$ for the same fields, but viewed as fiber-linear functions on the cotangent bundle of $G$ :

$$
P_{i}: T^{*} G \rightarrow \mathbb{R} ; P_{i}(g, p)=p\left(X_{i}(g)\right) .
$$

Given a choice of controls $u_{a}(t), a=1,2 \ldots, r$ not all identically zero, form the Hamiltonian

$$
H_{u}(g, p ; t)=\sum_{a=1}^{r} u_{a}(t) P_{a}(g, p) .
$$

The Maximum Principle [7, Theorem 12.1] asserts that a curve $\gamma$ in $G$ is singular for $V$ if and only if when we take its control $u$, and form the Hamiltonian $H_{u}$, then the corresponding Hamilton's equations have a nonzero solution $\zeta(t)=(q(t), p(t))$ that lies on the variety $P_{a}=0, a=1,2, \ldots, r$. Here 'Nonzero' means that $p(t) \neq 0$, for all $t$. The conditions $P_{a}=0$ mean that the solution lies in the annihilator of the distribution defined by $V$. The first of Hamilton's equations, implies that $\gamma$ has control $u$, so that the solution $\zeta$ does project onto $\gamma$ via the cotangent projection $\pi: T^{*} G \rightarrow G$.

The following two facts regarding symplectic geometry and Hamilton's equations allow us to immediately derive the Golé-Karidi form of the equations as expressed in Proposition 2.17. Fact 1. Hamilton's equations are equivalent to their 'Poisson form' $\dot{f}=\{f, H\}$. Here $f$ is an arbitrary smooth function on phase space, $\dot{f}=d f\left(X_{H}\right)$ is the derivative of $f$ along the Hamiltonian vector field $X_{H}$ for $H$, and $\{f, g\}$ is the Poisson bracket associated to the canonical symplectic form $\omega$, so that $\{f, g\}=\omega\left(X_{f}, X_{g}\right)$. Fact 2 . If $X$ is any vector field on $G$ (invariant or not), and if $P_{X}: T^{*} G \rightarrow \mathbb{R}$ denotes the corresponding fiber-linear function defined by $X$ as above, then $\left\{P_{X}, P_{Y}\right\}=-P_{[X, Y]}$.

Proof of Proposition 2.17 from the Maximum Principle. Take the $f=P_{i}$ and use, from Fact 2, that $\left\{P_{i}, P_{j}\right\}=$ $-\sum c_{i j}^{k} P_{k}$. The $P_{i}$ are equal to the $\eta_{i}$ of the proposition. 
Proposition 2.17 is just the coordinate form of Proposition 2.16, so we have also proved Proposition 2.16.

Proof of Corollary 2.14 from the Maximum Principle. Let $\gamma(t)$ be a singular extremal leaving the identity with control $u=\left(u_{1}, \ldots, u_{r}\right)$. Let $H_{u}$ be the time-dependent Hamiltonian generating the one-form $\zeta(t)$ along $\gamma$ as per the Maximum Principle. Since each of the $P_{i}$ are left-invariant, so is $H_{u}$. Now any left-invariant Hamiltonian $H_{u}$ on the cotangent bundle of a Lie group admits $n=\operatorname{dim}(G)$ 'constants' of motion - these being the $n$ components of the momentum map $J: T^{*} G \rightarrow \mathfrak{g}^{*}$ for the action of $G$ on itself by left translation. Recall that a 'constant of the motion' is a vector function that is constant along all the solutions to Hamilton's equations. Different solutions may have different constants. The momentum map in this situation is well-known to equal right-trivialization: $T^{*} G \rightarrow G \times \mathfrak{g}^{*}$ composed with projection onto the second factor. In other words, if $\zeta(t)$ is any solution for $H_{u}$, then $J(\zeta(t))=\lambda=$ const and also $J(\zeta(t))=\mathrm{d} R_{\gamma(t)}^{*} \zeta(t)$. Now, our $p(t)$ must annihilate $V_{\gamma(t)}$. The fact that $p(t)$ equals $\lambda$, right-translated along $\gamma$, and that $\Delta_{\gamma(t)}$ equals to $V=\Delta_{e}$, left-translated along $\gamma$ implies that $\lambda\left(\operatorname{Ad}_{\gamma(t)} V\right)=0$. We have established the claim.

\subsection{Abnormal varieties and connection with extremal polynomials}

The opportunity of considering the right-invariant trivialization of $T^{*} G$, hence arriving to Corollary 2.14, was suggested by the results of the two papers [14,15], where abnormal curves were characterized as those horizontal curves lying in specific algebraic varieties.

Given $\lambda \in \mathfrak{g}^{*} \backslash\{0\}$ we set

$$
Z^{\lambda}:=\left\{g \in G:\left(\left(\operatorname{Ad}_{g}\right)^{*} \lambda\right)_{\mid V}=0\right\} .
$$

In every Lie group the set $Z^{\lambda}$ is a proper real analytic variety. If $G$ is a nilpotent group, then $Z^{\lambda}$ is a proper real algebraic variety, which we call abnormal variety.

Proposition 2.21 (Restatement of Corollary 2.14). A horizontal curve $\gamma$ is abnormal if and only if $\gamma$ is contained in $Z^{\lambda}$ for some nonzero $\lambda \in \mathfrak{g}^{*}$.

We now prove that, in Carnot groups, the algebraic varieties $Z^{\lambda}$ coincide with the varieties introduced in $[14,15]$. This will follow from Proposition 2.22 below.

Let $e_{1}, \ldots, e_{n}$ be a basis of $\mathfrak{g}$ such that $e_{1}, \ldots, e_{r}$ is a basis of $V$. Let $X_{i}$ denote the extension of $e_{i}$ as a left-invariant vector field on $G$. Let $c_{i j}^{k}$ be the structure constants of $\mathfrak{g}$ in this basis, i.e.,

$$
\left[X_{i}, X_{j}\right]=\sum_{k} c_{i j}^{k} X_{k}
$$

For $\lambda \in \mathfrak{g}^{*}$, set

$$
P_{i}^{\lambda}(g):=\left(\left(\operatorname{Ad}_{g}\right)^{*} \lambda\right)\left(e_{i}\right) \text {. }
$$

Thus $Z^{\lambda}$ is the set of common zeros of the functions $P_{i}^{\lambda}, i=1, \ldots, r$. When $G$ is nilpotent, these functions are polynomials.

Proposition 2.22. Let $Y_{m}$ denote the extension of $e_{m}$ as a right-invariant vector field on $G$. Let $e_{1}^{*}, \ldots, e_{n}^{*}$ denote the basis vectors of $\mathfrak{g}^{*}$ dual to $e_{1}, \ldots, e_{n}$. For all $i, j=1, \ldots, n$, we have

$$
X_{i}=\sum_{m} P_{i}^{e_{m}^{*}} Y_{m}
$$

Moreover, the functions $P_{j}^{\lambda}$ satisfy $P_{j}^{\lambda}(e)=\lambda\left(e_{j}\right)$ and

$$
X_{i} P_{j}^{\lambda}=\sum_{k=1}^{n} c_{i j}^{k} P_{k}^{\lambda}, \quad \forall i, j=1, \ldots, n, \lambda \in \mathfrak{g}^{*} .
$$

In particular, in the setting of Carnot groups the functions $P_{j}^{\lambda}$ coincide with the extremal polynomials introduced in $[14,15]$. 
Proof. We verify (2.23) by

$$
\begin{aligned}
\sum_{m} P_{i}^{e_{m}^{*}}(g) Y_{m}(g) & =\sum_{m}\left(\operatorname{Ad}_{g}\right)^{*}\left(e_{m}^{*}\right)\left(e_{i}\right)\left(R_{g}\right)_{*} e_{m}=\sum_{m} e_{m}^{*}\left(\operatorname{Ad}_{g}\left(e_{i}\right)\right)\left(R_{g}\right)_{*} e_{m} \\
& =\left(R_{g}\right)_{*} \sum_{m} e_{m}^{*}\left(\operatorname{Ad}_{g}\left(e_{i}\right)\right) e_{m}=\left(R_{g}\right)_{*} \operatorname{Ad}_{g}\left(e_{i}\right)=\left(L_{g}\right)_{*} e_{i}=X_{i}(g) .
\end{aligned}
$$

Next, on the one hand, since $\left[X_{i}, Y_{j}\right]=0$,

$$
\left[X_{i}, X_{j}\right]=\sum_{m}\left(X_{i} P_{j}^{e_{m}^{*}}\right) Y_{m}
$$

On the other hand, from (2.23)

$$
\left[X_{i}, X_{j}\right]=\sum_{k} c_{i j}^{k} X_{k}=\sum_{m}\left(\sum_{k} c_{i j}^{k} P_{k}^{e_{m}^{*}}\right) Y_{m} .
$$

Thus

$$
X_{i} P_{j}^{e_{m}^{*}}=\sum_{k} c_{i j}^{k} P_{k}^{e_{m}^{*}}, \quad \forall i, j, m=1, \ldots, n .
$$

Formula (2.24) follows because, by definition, the functions $P_{j}^{\lambda}$ are linear in $\lambda$.

The extremal polynomials $\left(P_{j}^{v}\right)_{j=1, \ldots, n}^{v \in \mathbb{R}^{n}}$ were introduced in $[14,15]$ in the setting of Carnot groups; they were explicitly defined in a system of exponential coordinates of the second type associated to a basis of $\mathfrak{g}$ that is adapted to the stratification of $\mathfrak{g}$, see Section 2.2. Here, adapted simply means that the fixed basis $e_{1}, \ldots, e_{n}$ of $\mathfrak{g}$ consists of an (ordered) enumeration of a basis of the first layer $V_{1}$, followed by a basis of the second layer $V_{2}$, etc. It was proved in [15] that the extremal polynomials satisfy

$$
P_{j}^{v}(e)=v_{j} \quad \text { and } \quad X_{i} P_{j}^{v}=\sum_{k=1}^{n} c_{i j}^{k} P_{k}^{v} \quad \forall i, j=1, \ldots, n, \forall v \in \mathbb{R}^{n} .
$$

We need to check that, for any fixed $v \in \mathbb{R}^{n}$, the equality $P_{j}^{v}=P_{j}^{\lambda}$ holds for $\lambda:=\sum_{m} v_{m} e_{m}^{*}$. Indeed, the differences $Q_{j}:=P_{j}^{v}-P_{j}^{\lambda}$ satisfy

$$
Q_{j}(e)=0 \quad \text { and } \quad X_{i} Q_{j}=\sum_{k=1}^{n} c_{i j}^{k} Q_{k} \quad \forall i, j=1, \ldots, n .
$$

In particular, $X_{i} Q_{n}=0$ for any $i$ because, by the stratification assumption, $c_{i n}^{k}=0$ for any $i$, $k$. This implies that $Q_{n}$ is constant, i.e., that $Q_{n} \equiv 0$. We can then reason by reverse induction on $j$ and assume that $Q_{k} \equiv 0$ for any $k \geq j+1$; then, using the fact that $c_{i j}^{k}=0$ whenever $k \leq j$ (because the basis is adapted to the stratification), we have

$$
Q_{j}(e)=0 \quad \text { and } \quad X_{i} Q_{j}=\sum_{k=j+1}^{n} c_{i j}^{k} Q_{k}=0 \quad \forall i=1, \ldots, n .
$$

Hence also $Q_{j} \equiv 0$. This proves that $P_{j}^{v}=P_{j}^{\lambda}$, as desired.

Remark 2.25. In the study of Carnot groups of step 2 and step 3, it will be used that the varieties $W^{\lambda}$ defined below (which coincide with the abnormal varieties in the step-2 case) are subgroups. Namely, if $G$ is a Carnot group of step $s$ and highest layer $V_{s}$, and $\lambda \in \mathfrak{g}^{*}$, then the variety

$$
W^{\lambda}:=\left\{g \in G:\left(\left(\operatorname{Ad}_{g}\right)^{*} \lambda\right)_{\mid V_{s-1}}=0\right\}
$$

is a subgroup, whenever it contains the origin. Indeed, if $X \in \mathfrak{g}$ and $Y \in V_{s-1}$, then

$$
\left(\operatorname{Ad}_{\exp (X)}\right)^{*} \lambda(Y)=\left(e^{\operatorname{ad}_{X}}\right)^{*} \lambda(Y)=\lambda(Y+[X, Y]) .
$$


Hence, in exponential coordinates the set $W^{\lambda}$ is

$$
\left\{X \in \mathfrak{g}: \lambda(Y+[X, Y])=0, \forall Y \in V_{s-1}\right\}
$$

and, if it contains the origin, it is

$$
\left\{X \in \mathfrak{g}: \lambda([X, Y])=0, \forall Y \in V_{s-1}\right\} .
$$

Since the condition $\lambda([X, Y])=0$, for all $Y \in V_{s-1}$, is linear in $X$, we conclude that $W^{\lambda}$ is a subgroup.

\subsection{Lifts of abnormal curves}

Proposition 2.27 (Lifts of abnormal is abnormal). Let $\gamma:[0,1] \rightarrow G$ be a horizontal curve with respect to $V \subset \mathfrak{g}$. If there exists a Lie group $H$ and a surjective homomorphism $\pi: G \rightarrow H$ for which $\pi \circ \gamma$ is abnormal with respect to some $W \supseteq \mathrm{d} \pi_{e}(V)$, then $\gamma$ is abnormal.

Proof. Let $\operatorname{End}^{V}$ and End ${ }^{W}$ be the respective endpoint maps, as in the diagram below. For $u \in L^{2}([0,1], V)$ let $\pi_{*} u:=\mathrm{d} \pi_{e} \circ u$, which is an element in $L^{2}([0,1], W)$, because $\mathrm{d} \pi_{e}(V) \subseteq W$. Since $\pi$ is a group homomorphism, one can easily check that the following diagram commutes:

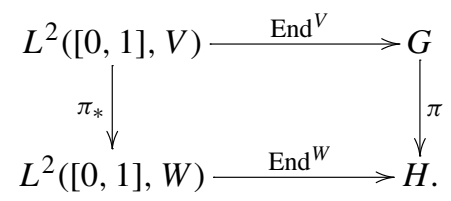

By assumption $\pi$ is surjective and so is $\mathrm{d} \pi_{g}$, for all $g \in G$. We conclude that $\mathrm{d} \operatorname{End}_{\pi_{*} u}^{W}$ is surjective, whenever $\mathrm{dEnd}_{u}^{V}$ is surjective.

Example 2.28 (Abnormal curves in a product). Let $G$ and $H$ be two Lie groups. Let $V \subset \operatorname{Lie}(G)$ and $W \subset \operatorname{Lie}(H)$. Assume that $W \neq \operatorname{Lie}(H)$. Let $\gamma:[0,1] \rightarrow G \times H$ be a curve. If $\gamma=\left(\gamma_{1}(t), e\right)$ with $\gamma_{1}:[0,1] \rightarrow G$ horizontal with respect to $V$, then $\gamma$ is abnormal with respect to $V \times W$. Indeed, this fact is an immediate consequence of Proposition 2.27 using the projection $G \times H \rightarrow H$ and the fact that the constant curve in $H$ is abnormal with respect to the proper subspace $W$.

Remark 2.29. Let $G$ and $H$ be two Lie groups. If $\gamma_{1}:[0,1] \rightarrow G$ is not abnormal with respect to some $V \subset \operatorname{Lie}(G)$ and $\gamma_{2}:[0,1] \rightarrow H$ is not abnormal with respect to some $W \subset \operatorname{Lie}(H)$, then $\left(\gamma_{1}, \gamma_{2}\right):[0,1] \rightarrow G \times H$ is not abnormal with respect to $V \times W$.

Example 2.30 $(H \times H)$. Let $H$ be the Heisenberg group equipped with its contact structure. By Example 2.28 and Remark 2.29, the abnormal curves leaving from the origin in $H \times H$ are the curves of the form $(\gamma(t), e)$ or $(e, \gamma(t))$, where $\gamma:[0,1] \rightarrow H$ is any horizontal curve. In particular, $\operatorname{Abn}(e)=H \times\{e\} \cup\{e\} \times H$, which has codimension 3 .

\subsection{Normal curves}

Let $(G, V)$ be a polarized group such that $V$ is bracket generating. Equipping $V$ with a scalar product $\|\cdot\|_{2}$, we get a left-invariant sub-Riemannian structure on $G$. Recall that from Pontrjagin Maximum Principle any curve that is length minimizing with respect to the sub-Riemannian distance is either abnormal, or normal (in the sense that we now recall), or both normal and abnormal. A curve $\gamma$ with control $u$ is normal if there exist $\lambda_{0} \neq 0$ and $\lambda_{1} \in T_{\gamma(1)}^{*} G$ such that $\left(\lambda_{0}, \lambda_{1}\right)$ vanishes on the image of the differential at $u$ of the extended endpoint map $\widetilde{\text { End }}: L^{2}([0,1], V) \rightarrow \mathbb{R} \times G$, $v \mapsto\left(\|v\|_{2}, \operatorname{End}(v)\right)$. Let $\operatorname{Abn}^{n o r}(e)$ denote the set of points connected to the origin by curves which are both normal and abnormal. Let $\mathrm{Abn}^{l m}(e)$ denote the set of points connected to the origin by abnormal curves that are locally length minimizing with respect to the sub-Riemannian distance. 
Lemma 2.31. Let $G$ be a polarized Lie group. The Sard Property holds for normal abnormals. Namely, the set $\operatorname{Abn}^{n o r}(e)$ is contained in a sub-analytic set of codimension at least 1.

Proof. We will make use of the sub-Riemannian exponential map, see [1]. Namely, normal curves starting from $e$ have cotangent lifts which satisfy a Hamiltonian equation. Solving this equation with initial datum $\xi \in T_{e}^{*} G$ defines a control $\widetilde{\operatorname{Exp}}(\xi) \in L^{2}([0,1], V)$. Composing with the endpoint map, one gets the sub-Riemannian exponential map $\operatorname{Exp}: T_{e}^{*} G \rightarrow G$,

$$
\operatorname{Exp}=\text { End } \circ \widetilde{\operatorname{Exp}} \text {. }
$$

Points in $\operatorname{Abn}^{n o r}(e)$ are values of Exp where the differential of End is not onto. Therefore, they are singular values of Exp. Since Exp is analytic, the set of its singular points is analytic, thus the set of its singular values is a sub-analytic subset of $G$. By Sard's theorem, it has measure zero, therefore its codimension is at least 1 .

\subsection{The Goh condition}

Let $(G, V)$ be a polarized group as in Section 2.7. We introduce the well-known Goh condition by using the formalism of Corollary 2.14 .

Definition 2.32. We say that an abnormal curve $\gamma:[0,1] \rightarrow G$ leaving from the origin $e$ satisfies the Goh condition if there exists $\lambda \in \mathfrak{g}^{*} \backslash\{0\}$ such that

$$
\lambda\left(\operatorname{Ad}_{\gamma(t)}(V+[V, V])\right)=0 \quad \text { for every } t \in[0,1] .
$$

Equivalently, $\gamma$ satisfies the Goh condition if and only if there exists a right-invariant 1-form $\alpha$ on $G$ such that $\alpha\left(\Delta_{\gamma(t)}^{2}\right)=\{0\}$ for every $t \in[0,1]$, where $\Delta^{2}$ is the left-invariant distribution induced by $V+[V, V]$. Equivalently, denoting by $u$ the controls associated with $\gamma$ and recalling Proposition 2.3, if and only if the space

$$
\bigcup_{t \in[0,1]} \operatorname{Ad}_{\gamma(t)}(V+[V, V])=d R_{\gamma(1)}^{-1}\left(\operatorname{Im}\left(\operatorname{dEnd}_{u}\right)\right)+\bigcup_{t \in[0,1]} \operatorname{Ad}_{\gamma(t)}([V, V])
$$

is a proper subspace of $\mathfrak{g}=T_{e} G$, which a posteriori is contained in $\operatorname{ker} \lambda$, for $\lambda$ as in (2.33).

Remark 2.35. Clearly, any $\lambda$ such that (2.33) holds is in the annihilator of $V+[V, V]$, just by considering $t=0$ in (2.33).

The importance of the Goh condition stems from the following well-known fact: if $\gamma$ is a strictly abnormal length minimizer (i.e., a length minimizer that is abnormal but not also normal), then it satisfies Goh condition for some $\lambda \in \mathfrak{g}^{*} \backslash\{0\}$. See [7, Chapter 20] and also [6]. Notice that not necessarily all the $\lambda$ 's as in (2) of Corollary 2.14 will satisfy (2.33), but at least one will. On the contrary, in the particular case $\operatorname{dim} V=2$, every abnormal curve satisfies the Goh condition for every $\lambda$ as in Corollary 2.14(2); see Remark 2.8 and (2.9) in particular.

\section{Step-2 Carnot groups}

\subsection{Facts about abnormal curves in two-step Carnot groups}

We want to study the abnormal set $\operatorname{Abn}(e)$ defined in (2.13) with the use of the abnormal varieties defined in (2.20). In fact, by Proposition 2.21 we have the inclusion

$$
\operatorname{Abn}(e) \subseteq \bigcup_{\lambda \in \mathfrak{g}^{*} \backslash\{0\} \text { s.t. } e \in Z^{\lambda}} Z^{\lambda}
$$

In this section we will consider the case when the polarized group $(G, V)$ is a Carnot group of step 2. Fix an element $\lambda \in \mathfrak{g}^{*}$. Since $\mathfrak{g}^{*}=V_{1}^{*} \oplus V_{2}^{*}$, we can write $\lambda=\lambda_{1}+\lambda_{2}$ with $\lambda_{i} \in V_{i}^{*}$. As noticed in Remark 2.25, since $G$ has step 2, if $X \in \mathfrak{g}$ and $Y \in V_{1}$, then 


$$
\left(\operatorname{Ad}_{\exp (X)}\right)^{*} \lambda(Y)=\left(e^{\operatorname{ad}_{X}}\right)^{*} \lambda(Y)=\lambda_{1}(Y)+\lambda_{2}([X, Y]) .
$$

Notice that, if $e=\exp (0) \in Z^{\lambda}$, then $\lambda_{1}(Y)=0$ for all $Y \in V_{1}$. Thus $\lambda_{1}=0$. Therefore, any variety $Z^{\lambda}$ containing the identity is of the form

$$
Z^{\lambda}=Z^{\lambda_{2}}=\exp \left\{X \in \mathfrak{g}: \lambda_{2}([X, Y])=0 \forall Y \in V_{1}\right\} .
$$

The condition

$$
\lambda_{2}([X, Y])=0, \quad \forall Y \in V_{1},
$$

is linear in $X$, hence the set

$$
\mathfrak{z}^{\lambda}:=\log \left(Z^{\lambda}\right)=\left\{X \in \mathfrak{g}: \lambda_{2}([X, Y])=0 \forall Y \in V_{1}\right\}
$$

is a vector subspace.

If now $\gamma$ is a horizontal curve contained in $Z^{\lambda}$ (and hence abnormal) with $\gamma(0)=e$, then $\gamma$ is contained in the subgroup $H^{\lambda}$ generated by $\mathfrak{z}^{\lambda} \cap V_{1}$, i.e.,

$$
H^{\lambda}:=\exp \left(\left(\mathfrak{z}^{\lambda} \cap V_{1}\right) \oplus\left[\mathfrak{z}^{\lambda} \cap V_{1}, \mathfrak{z}^{\lambda} \cap V_{1}\right]\right) .
$$

This implies that

$$
\operatorname{Abn}(e) \subseteq \bigcup_{\lambda \in \mathfrak{g}^{*} \backslash\{0\}, \lambda_{1}=0} H^{\lambda} .
$$

It is interesting to notice that also the reverse inclusion holds: indeed, for any $\lambda \in \mathfrak{g}^{*} \backslash\{0\}$ with $\lambda_{1}=0$ and any point $p \in H^{\lambda}$, there exists an horizontal curve $\gamma$ from the origin to $p$ that is entirely contained in $H^{\lambda} ; \gamma$ is then contained in $Z^{\lambda}$ and hence it is abnormal by Proposition 2.21. We deduce that

$$
\operatorname{Abn}(e)=\bigcup_{\lambda \in \mathfrak{g}^{*} \backslash\{0\}, \lambda_{1}=0} H^{\lambda} .
$$

We are now ready to prove a key fact in the setting of two-step Carnot groups: every abnormal curve is not abnormal in some subgroup. We first recall that a Carnot subgroup in a Carnot group is a Lie subgroup generated by a subspace of the first layer.

Lemma 3.3. Let $G$ be a 2-step Carnot group. For each abnormal curve $\gamma$ in $G$, there exists a proper Carnot subgroup $G^{\prime}$ of $G$ containing $\gamma$, in which $\gamma$ is a non-abnormal horizontal curve.

Proof. Let $G^{\prime}$ be the smallest Carnot subgroup of $G$ containing $\gamma$. If by contradiction $\gamma$ is abnormal in $G^{\prime}$, then there exists $\lambda \in\left(\mathfrak{g}^{\prime}\right)^{*} \backslash\{0\}$, with $\lambda_{1}=0$, such that $\gamma \subset H^{\lambda}$, where $H^{\lambda}$ is the subgroup of $G^{\prime}$ defined in (3.1), which is proper since $\lambda \neq 0$. We conclude that $G^{\prime}$ is not minimal.

\subsection{Parameterizing abnormal varieties within free two-step Carnot groups}

Let $G$ be a free-nilpotent 2-step Carnot group. Let $m \leq r:=\operatorname{dim}\left(V_{1}\right)$. Fix an $m$-dimensional vector subspace $W_{m}^{\prime} \subset V_{1}$. Denote by $G_{m}$ the subgroup generated by $W_{m}^{\prime}$, and $X_{m}=G L(r, \mathbb{R}) \times G_{m}$, equipped with the left-invariant distribution given at the origin by $W_{m}:=\{0\} \oplus W_{m}^{\prime}$. Observe that $G L(r, \mathbb{R})$ acts on $G$ by graded automorphisms. Let

$$
\Phi_{m}: X_{m} \rightarrow G, \quad(g, h) \mapsto g(h) .
$$

In a polarized group $(X, V)$, given a submanifold $Y \subset X$, the endpoint map relative to $Y$ is $\operatorname{End}^{Y}: Y \times L^{2}([0,1], V) \rightarrow$ $X,(y, u) \mapsto \gamma_{u}^{(y)}(1)$, where $\gamma_{u}^{(y)}$ satisfies (2.1) with $\gamma_{u}^{(y)}(0)=y$. We say that a horizontal curve $\gamma$ with control $u$ is non-singular relative to $Y$ if the differential at $(\gamma(0), u)$ of the endpoint map relative to $Y$ is onto.

Lemma 3.4. Let $G$ be a free 2-step Carnot group of rank $r$. For every abnormal curve $\gamma$ in $G$, there exists an integer $m<r$ and a horizontal curve $\sigma$ in $X_{m}$ such that $\Phi_{m}(\sigma)=\gamma$, and $\sigma$ is non-singular relative to $\Phi_{m}^{-1}(e)$. 
Proof. Let $\gamma$ be an abnormal curve in $G$ starting at $e$, with control $u$. By Lemma 3.3, $\gamma$ is contained in the Carnot subgroup $G^{\prime}$ of $G$ generated by some subspace $V_{1}^{\prime} \subset V_{1}$ and is not abnormal in $G^{\prime}$. Let $m=\operatorname{dim}\left(V_{1}^{\prime}\right)$. Then there exists $g \in G L(r, \mathbb{R})$ such that $V_{1}^{\prime}=g\left(W_{m}^{\prime}\right)$, and thus $G^{\prime}=g\left(G_{m}\right)$. Let $\sigma=\left(g, g^{-1}(\gamma)\right)$. This is a horizontal curve in $X_{m}$. Consider the endpoint map on $X_{m}$ relative to the submanifold $\Phi_{m}^{-1}(e)=G L(r, \mathbb{R}) \times\{e\}$. Since $\gamma$ is not abnormal in $G^{\prime}$, the image $I$ of the differential at $\left((g, e), g^{-1}(u)\right)$ of the endpoint map contains $\{0\} \oplus T_{g^{-1}(\gamma(1))} G_{m}$. Every curve of the form $t \mapsto\left(k, g^{-1}(\gamma(t))\right)$ with fixed $k \in G L(r, \mathbb{R})$ is horizontal, so $I$ contains $T_{g}(G L(r, \mathbb{R})) \oplus\{0\}$. One concludes that $I=T_{\left(g, g^{-1} \gamma(1)\right)} X_{m}$, i.e., $\sigma$ is non-singular relative to $\Phi_{m}^{-1}(e)$. By construction, $\Phi_{m}(\sigma)=\gamma$.

\subsection{Application to general 2-step Carnot groups}

Proposition 3.5. Let $G$ be a 2-step Carnot group. There exists a proper algebraic set $\Sigma \subset G$ that contains all abnormal curves leaving from the origin.

Proof. Let $f: \tilde{G} \rightarrow G$ be a surjective homomorphism from a free 2-step Carnot group of the same rank as $G$. Let $\gamma$ be an abnormal curve leaving from the origin in $G$. It has a (unique) horizontal lift $\tilde{\gamma}$ in $\tilde{G}$ leaving from the origin. According to Lemma 3.4, there exists an integer $m$ and a non-singular (relative to $\Phi_{m}^{-1}(e)$ ) horizontal curve $\sigma$ in $X_{m}$ such that $\Phi_{m}(\sigma)=\tilde{\gamma}$, i.e., $f \circ \Phi_{m}(\sigma)=\gamma$. Namely, there exists $g \in G L(r, \mathbb{R})$ such that $\sigma(t)=\left(g, g^{-1} \tilde{\gamma}(t)\right)$. Consider the endpoint map $\operatorname{End}^{Y}$ on $X_{m}$ relative to the submanifold $Y:=\Phi_{m}^{-1}(e)$. Let us explain informally the idea of the conclusion of the proof. The composition $f \circ \Phi_{m} \circ$ End $^{Y}$ is an endpoint map for $G$, with starting point at the identity $e$. Hence, since the differential of End ${ }^{Y}$ at the control of $\sigma$ is onto, but the differential of $f \circ \Phi_{m} \circ$ End $^{Y}$ is not, the point $\gamma(1)$ is a singular value of $f \circ \Phi_{m}$. Hence, we will conclude using Sard's theorem.

Let us now give a more formal proof of the last claims. Consider the map $\phi_{m}: Y \times L^{2}\left([0,1], W_{m}\right) \rightarrow L^{2}\left([0,1], V_{1}\right)$, defined as $\left(\phi_{m}(g, u)\right)(t):=g(u(t)) \in V_{1} \subseteq T_{e} \tilde{G}$, for $t \in[0,1]$. We then point out the equality

$$
f \circ \Phi_{m} \circ \operatorname{End}^{Y}=\text { End } \circ f_{*} \circ \phi_{m},
$$

where End : $L^{2}\left([0,1], V_{1}\right) \rightarrow G$ is the endpoint map of $G$ and $f_{*}: L^{2}\left([0,1], V_{1}\right) \rightarrow L^{2}\left([0,1], V_{1}\right)$ is the map

$$
\left(f_{*}(u)\right)(t)=(\mathrm{d} f)_{e}(u(t)) \in V_{1} \subseteq T_{e} G .
$$

Since $\sigma$ is abnormal, i.e., the differential $\mathrm{dEnd}_{u_{\gamma}}$ is not surjective, and the differential of $\operatorname{End}^{Y}$ at the point $\left(g, u_{\sigma}\right)=$ $\left(f_{*} \circ \phi_{m}\right) u_{\gamma}$ is surjective, from (3.6) we deduce that $\gamma(1)$ is a singular value for $f \circ \Phi_{m}$. By the classical Sard Theorem, the set $\Sigma_{m}$ of singular values of $f \circ \Phi_{m}$ has measure 0 in $G$. So has the union $\tilde{\Sigma}:=\cup_{m=1}^{r-1} \Sigma_{m}$ of these sets. By Tarski-Seidenberg's theorem [8, Proposition 2.2.7], $\tilde{\Sigma}$ is a semi-algebraic set, since the map $f \circ \Phi_{m}$ is algebraic and the set of critical points of an algebraic map is an algebraic set. Moreover, from [8, Proposition 2.8.2] we have that this semi-algebraic set is contained in an algebraic set $\Sigma$ of the same dimension. Since $\tilde{\Sigma}$ has measure zero, the set $\Sigma$ is a proper algebraic set.

Example 3.7 (Abnormal curves not lying in any proper subgroup). Key to our proof was the property, encoded in Equation (3.1), that every abnormal curve is contained in a proper subgroup of $G$. This property typically fails for Carnot groups of step greater than 2. Golé and Karidi [11] constructed a Carnot group of step 4 and rank 2 for which this property fails: namely, there is an abnormal curve that is not contained in any proper subgroup of their group. Further on in this paper (Section 6.3) we show that this property fails for the free 3-step rank-3 Carnot group.

\subsection{Codimension bounds on free 2-step Carnot groups}

In this section we prove Theorem 1.4; we will make extensive use of the result and notation of Section 3.1. In the sequel, we denote by $G$ a fixed free Carnot group of step 2 and by $r=\operatorname{dim} V_{1}$ its rank.

We identify $G$ with its Lie algebra, which has the form $V \oplus \Lambda^{2} V$ for $V=V_{1} \cong \mathbb{R}^{r}$ a real vector space of dimension $r$. The Lie bracket is $[(v, \xi),(w, \eta)]=(0, v \wedge w)$. When we use the exponential map to identify the group with its Lie algebra, the equation for a curve $(x(t), \xi(t))$ to be horizontal reads

$$
\dot{x}=u, \quad \dot{\xi}=x \wedge u .
$$

If $W \subset V$ is a subspace, then the group it generates has the form $W \oplus \Lambda^{2} W \subset V \oplus \Lambda^{2} V$. 


\subsection{Proof that $\operatorname{Abn}(e)$ is contained in a set of codimension $\geq 3$}

We use the view point discussed in Section 3.1 where we defined the sets $\mathfrak{z}^{\lambda}$ and $H^{\lambda}$. We first claim that

$$
\operatorname{dim} \mathfrak{z}^{\lambda} \cap V=\operatorname{dim}\left\{X \in V: \lambda_{2}([X, Y])=0 \forall Y \in V\right\} \leq r-2,
$$

for any $\lambda \in \mathfrak{g}^{*} \backslash\{0\}$ such that $\lambda_{1}=0$. Indeed, since $\lambda_{2} \neq 0$, the alternating 2-form $w(\lambda):(X, Y) \mapsto \lambda_{2}([X, Y])$ has rank at least 2 . (3.2),

Then, by (3.8), each $\mathfrak{z}^{\lambda} \cap V$ is contained in some $W \subset V$ with $\operatorname{dim}(W)=r-2$, hence $H^{\lambda} \subseteq W \oplus \Lambda^{2} W$ and, by

$$
\operatorname{Abn}(e)=\bigcup_{\lambda \in \mathfrak{g}^{*} \backslash\{0\}, \lambda_{1}=0} H^{\lambda} \subseteq \bigcup_{W \in \operatorname{Gr}(r, r-2)} W \oplus \Lambda^{2} W .
$$

In fact, the equality

$$
\operatorname{Abn}(e)=\bigcup_{W \in G r(r, r-2)} W \oplus \Lambda^{2} W
$$

holds: this is because every codimension 2 subspace $W \subset V$ is the kernel of a rank 2 skew-symmetric 2-form (the pull-back of a nonzero form on the 2-dimensional space $V / W$ ), and every such skew-symmetric form corresponds to a covector $\lambda_{2} \in V_{2}^{*}=\Lambda^{2} V^{*}$.

We now notice that the Grassmannian $G r(r, r-2)$ of $(r-2)$-dimensional planes in $V$ has dimension $2(r-2)$ and that each $W \oplus \Lambda^{2} W$ is (isomorphic to) the free group $\mathbb{F}_{m, 2}$ of rank $m=r-2$ and step 2, i.e.,

$$
\operatorname{dim}\left(W \oplus \Lambda^{2} W\right)=m+\frac{m(m-1)}{2}=\frac{(r-1)(r-2)}{2} .
$$

It follows that the set $\cup_{W \in G r(r, r-2)} W \oplus \Lambda^{2} W$ can be parametrized with a number of parameters not greater than

$$
\operatorname{dim} \mathbb{F}_{m, 2}+\operatorname{dim} \operatorname{Gr}(r, m)=r(r+1) / 2-3 .
$$

Since $\operatorname{dim} G=r(r+1) / 2$, the codimension 3 of Theorem 1.4 follows from (3.9).

\subsection{Proof that $\operatorname{Abn}(e)$ is a semialgebraic set of codimension $\geq 3$}

Let $k=\lfloor(r-2) / 2\rfloor$ and let $W$ be a codimension 2 vector subspace of $V_{1}$. Every pair $(\xi, \eta) \in W \oplus \Lambda^{2} W$ can be written as

$$
\xi=\sum_{j=1}^{r-2} x_{j} \xi_{j}, \quad \eta=\sum_{i=1}^{k} z_{i} \xi_{2 i-1} \wedge \xi_{2 i}
$$

for some $(r-2)$-uple of vectors (e.g., a basis) $\left(\xi_{j}\right)_{1 \leq j \leq r-2}$ of $W$. Conversely, every pair $(\xi, \eta) \in \mathfrak{g}=V \oplus \Lambda^{2} V$ of this form belongs to $W \oplus \Lambda^{2} W$ for some codimension 2 subspace $W$ of $V_{1}$. Therefore

$$
\bigcup_{W \in G r(r, r-2)} W \oplus \Lambda^{2} W
$$

is the projection on the first factor of the algebraic subset

$$
\left\{\left(\xi, \eta, \xi_{1}, \ldots, \xi_{r-2}, x_{1}, \ldots, x_{r-2}, z_{1}, \ldots, z_{k}\right): \xi=\sum_{j=1}^{r-2} x_{j} \xi_{j}, \eta=\sum_{i=1}^{k} z_{i} \xi_{2 i-1} \wedge \xi_{2 i}\right\}
$$

of $\mathfrak{g} \times V^{r-2} \times \mathbb{R}^{r-2} \times \mathbb{R}^{k}$. Since the exponential map is an algebraic isomorphism, $\operatorname{Abn}(e)=\bigcup_{W \in G r(r, r-2)} W \oplus \Lambda^{2} W$ is semi-algebraic, and it is contained in an algebraic set of the same codimension (see [8, Proposition 2.8.2]).

In the rest of this section we proceed with the more precise description of the set $\mathrm{Abn}(e)$, as described in Theorem 1.4.

Each $\xi \in \Lambda^{2} V$ can be viewed, by contraction, as a linear skew symmetric map $\xi: V^{*} \rightarrow V$. For example, if $\xi=v \wedge w$, then this map sends $\alpha \in V^{*}$ to $\alpha(v) w-\alpha(w) v$. 
Definition 3.10. For $\xi \in \Lambda^{2} V$ let $\operatorname{supp}(\xi) \subset V$ denote the image of $\xi$, when $\xi$ is viewed as a linear map $V^{*} \rightarrow V$. For $(v, \xi) \in V \oplus \Lambda^{2} V \operatorname{set} \operatorname{supp}(v, \xi)=\mathbb{R} v+\operatorname{supp}(\xi)$. Finally, set $\operatorname{rank}(v, \xi)=\operatorname{dim}(\operatorname{supp}(v, \xi))$.

Proposition 3.11. If $G$ is the free 2-step nilpotent group on $r$ generators then

$$
\operatorname{Abn}(e)=\{(v, \xi): \operatorname{rank}(v, \xi) \leq r-2\} .
$$

Proof. From (3.9) we can directly derive the new characterization. Suppose that $W \subset V$ is any subspace and $(w, \xi) \in$ $W \oplus \Lambda^{2} W$. Then clearly $\operatorname{supp}(w, \xi) \subset W$. Conversely, if $(w, \xi)$ has support a subspace of $W$, then one easily checks that $(w, \xi) \in W \oplus \Lambda^{2} W$. Taking $W$ an arbitrary subspace of rank $r-2$ the result follows.

By combining Proposition 3.11 with some linear algebra we will conclude the proof of Theorem 1.4. This proof is independent of Sections 3.5 and 3.6 and yields a different perspective on the abnormal set.

Proof of Theorem 1.4. Let $G$ be the free-nilpotent 2-step group on $r$ generators. First, we write the polynomials defining $\operatorname{Abn}(e)$, then we compute dimensions. It is simpler to divide up into the case of even and odd rank $r$. We will consider the case of even rank in detail and leave most of the odd rank case up to the reader.

The linear algebraic Darboux theorem will prove useful for computations. All bivectors have even rank. This theorem asserts that the bivector $\xi \in \Lambda^{2} V$ has rank $2 m$ if and only if there exists $2 m$ linearly independent vectors $e_{1}, f_{1}, e_{2}, f_{2}, \ldots e_{m}, f_{m}$ in $V$ such that $\xi=\sum_{i=1}^{m} e_{i} \wedge f_{i}$.

Let us now specialize to the case where $r=\operatorname{dim}(V)$ is even. Write

$$
r=2 s .
$$

Using Darboux one checks that $\operatorname{rank}(0, \xi) \leq r-2$ if and only if $\xi^{s}=0$ (written out in components, $\xi$ is a skewsymmetric $2 r \times 2 r$ matrix and the vanishing of $\xi^{s}$ is exactly the vanishing of the Pfaffian of this matrix). Now, if $\operatorname{rank}(0, \xi)=r-2$ and $\operatorname{rank}(v, \xi) \leq r-2$, it must be the case that $v \in \operatorname{supp}(\xi)$; equivalently, in the Darboux basis, $v=\sum_{i=1}^{m} a_{i} e_{i}+\sum_{i=1}^{m} b_{i} f_{i}$. It follows in this case that $v \in \operatorname{supp}(\xi)$ if and only if $v \wedge \xi^{s-1}=0$. Now, if $\operatorname{rank}(0, \xi)<r-2$ then $\operatorname{rank}(0, \xi) \leq r-4$ and so $\operatorname{rank}(v, \xi) \leq r-3$ for any $v \in V$. But $\operatorname{rank}(0, \xi)<r-2$ if and only if $\xi^{s-1}=0$ in which case automatically $v \wedge \xi^{s-1}=0$.

We have proven that in the case $r=2 s$, the equations for $\operatorname{Abn}(e)$ are the polynomial equations $\xi^{s}=0$ and $v \wedge$ $\xi^{s-1}=0$.

To compute dimension, we stratify $\operatorname{Abn}(e)$ according to the rank of its elements. The dimensions of the strata are easily checked to decrease with decreasing rank, so that the dimension of $\operatorname{Abn}(e)$ equals the dimension of the largest stratum, the stratum consisting of the $(v, \xi)$ of even rank $r-2$. (The Darboux theorem and a bit of work yields that the stratum having rank $k$ with $k$ odd consists of exactly one $G l(V)$ orbit while the stratum having rank $k$ with $k$ even consists of exactly two $G l(V)$ orbits.) A point $(v, \xi)$ is in this stratum if and only if $\xi^{s}=0$ while $\xi^{s-1} \neq 0$ and $v \in \operatorname{supp}(\xi)$. Let us put the condition on $v$ aside for the moment. The first condition on $\xi$ is the Pfaffian equation which defines an algebraic hypersurface in $\Lambda^{2} V$, the zero locus of the Pfaffian of $\xi$. The second equation for $\xi$ defines the smooth locus of the Pfaffian. Thus, the set of $\xi$ 's satisfying the first two equations has dimension 1 less than that of $\Lambda^{2} V$, so its dimension is $\left(\begin{array}{c}r \\ 2\end{array}\right)-1$. Now, on this smooth locus $\{P f=0\}_{\text {smooth }} \subset\{P f=0\}$ we have a well-defined algebraic map $F:\{P f=0\}_{\text {smooth }} \rightarrow G r(r, r-2)$ which sends $\xi$ to $F(\xi)=\operatorname{supp}(\xi)$. Let $U \rightarrow G r(r, r-2)$ denote the canonical rank $r-2$ vector bundle over the Grassmannian. Thus $U \subset \mathbb{R}^{r} \times G r(r, r-2)$ consists of pairs $(v, P)$ such that $v \in P$. Then $F^{*} U$ is a rank $r-2$ vector bundle over $\{P f=0\}_{\text {smooth }}$ consisting of pairs $(v, \xi) \in \mathbb{R}^{2} \times \Lambda^{2} V$ such that $v \in \operatorname{supp}(\xi)$ and $\xi$ has rank $r-2$. In other words, the additional condition $v \in \operatorname{supp}(\xi)$ says exactly that $(v, \xi) \in F^{*} U$. It follows that the dimension of this principle stratum is $\operatorname{dim}\left(F^{*} U\right)=\left(\left(\begin{array}{c}r \\ 2\end{array}\right)-1\right)+(r-2)=\operatorname{dim}(G)-3$.

Regarding the odd rank case

$$
r=2 s+1
$$

the same logic shows that the equations defining $\operatorname{Abn}(e)$ are $\xi^{s}=0$ and involves no condition on $v$. A well-known matrix computation [5] shows that the subvariety $\left\{\xi^{s}=0\right\}$ in the odd rank case has codimension 3. Since the map $V \oplus \Lambda^{2} V \rightarrow \Lambda^{2} V$ is a projection, and since $\operatorname{Abn}(e)$ is the inverse image of $\left\{\xi^{s}=0\right\} \subset \Lambda^{2} V$ under this projection, its image remains codimension 3 . 
Recall that the rank of $\xi \in \Lambda^{2} V$ is the (even) dimension $d$ of its support. For an open dense subset of elements of $\Lambda^{2} V$, the rank is as large as possible: $r$ if $r$ is even and $r-1$ if $r$ is odd. We call singular the elements $\xi \in \Lambda^{2} V$ whose rank is less than the maximum and we write $\left(\Lambda^{2} V\right)_{\text {sing }}$ to denote the set of singular elements. From Proposition 3.11 we easily deduce the following.

Proposition 3.12. The projection of $\operatorname{Abn}(e)$ onto $\Lambda^{2} V$ coincides with the singular elements $\left(\Lambda^{2} V\right)_{\operatorname{sing}} \subset \Lambda^{2} V$.

Remark 3.13. A consequence of the previous result is the fact that elements of the form $(0, \xi)$ where $\operatorname{rank}(\xi)$ is maximal can never be reached by abnormal curves. Notice that such elements are in the center of the group.

To be more precise about $\operatorname{Abn}(e)$ we must divide into two cases according to the parity of $r$.

Theorem 3.14. If $G=V \oplus \Lambda^{2} V$ is a free Carnot group with odd rank $r$, then $\operatorname{Abn}(e)=V \oplus\left(\Lambda^{2} V\right)_{\text {sing. }}$.

The previous result, as well as the following one, easily follows from Proposition 3.11. To describe the situation for $r$ even, let us write $\left(\Lambda^{2} V\right)_{d}$ for those elements of $\Lambda^{2} V$ whose rank is exactly $d$ and $\left(\Lambda^{2} V\right)_{<d}$ for those elements whose rank is strictly less than $d$.

Theorem 3.15. If $G=V \oplus \Lambda^{2} V$ is a free Carnot group with even rank $r$, then $\operatorname{Abn}(e)$ is the union $Y \cup Y_{1}$ of the two quasiprojective subvarieties

$$
\begin{aligned}
& Y=\left\{(v, \xi) \in V \oplus \Lambda^{2} V: v \in \operatorname{supp}(\xi), \xi \in\left(\Lambda^{2} V\right)_{r-2}\right\} \\
& Y_{1}=V \times\left(\Lambda^{2} V\right)_{<r-2} .
\end{aligned}
$$

In particular, $\operatorname{Abn}(e)$ is a singular algebraic variety of codimension 3.

We observe that $Y_{1}=\bar{Y} \backslash Y$.

Remark 3.16. Given any $g=(v, \xi) \in G$ we can define its singular rank to be the minimum of the dimensions of the image of the differential of the endpoint map $d \operatorname{End}(\gamma)$, where the minimum is taken over all $\gamma$ that connect 0 to $g$. Thus, the singular rank of $g=0$ is $r$ and is realized by the constant curve, while if $\xi$ is generic then the singular rank of $g=(0, \xi)$ is $\operatorname{dim}(G)$, which means that every horizontal curve connecting 0 to $g$ is not abnormal.

It can be easily proved that, if $r$ is even and $v \in \operatorname{supp}(\xi)$, then the singular rank of $g$ is just $\operatorname{rank}(\xi)$. In this case we take a $\lambda$ with $\operatorname{ker}(\lambda)=\operatorname{supp}(\xi)$ and realize $g$ by any horizontal curve lying inside $G(\lambda)$.

\section{Sufficient condition for Sard's property}

In Section 2.1 we observed that, given a polarized group $(G, V)$ and a horizontal curve $\gamma$ such that $\gamma(0)=e$ and with control $u$, the space $\left(\mathrm{d} R_{\gamma(1)}\right)_{e} V+\left(\mathrm{d} L_{\gamma(1)}\right)_{e} V+\mathcal{S}(\gamma(1))$ is a subset of $\operatorname{Im}\left(\mathrm{dEnd}_{u}\right) \subset T_{\gamma(1)} G$. Therefore, if $g \in G$ is such that

$$
\operatorname{Ad}_{g^{-1}} V+V+\left(\mathrm{d} L_{g}\right)^{-1} \mathcal{X}(g)=\mathfrak{g},
$$

for some subset $\mathcal{X}$ of $\mathcal{S}$, then $g$ is not a singular value of the endpoint map. Here we denoted with $\mathcal{X}(g)$ the space of vector fields in $\mathcal{X}$ evaluated at $g$. In particular, if the equation above is of polynomial type (resp. analytic), then $(G, V)$ has the Algebraic (resp. Analytic) Sard Property.

In the following we embed both sides of (4.1) in a larger Lie algebra $\tilde{\mathfrak{g}}$, and we find conditions on $\tilde{\mathfrak{g}}$ that are sufficient for (4.1) to hold. The idea is to consider a group $\tilde{G}$ that acts, locally, on $G$ via contact mappings, that is, diffeomorphisms that preserve the left-invariant subbundle $\Delta$. It turns out that the Lie algebra $\tilde{\mathfrak{g}}$ of $\tilde{G}$, viewed as algebra of left-invariant vector fields on $\tilde{G}$, represents a space of contact vector fields of $G$. 


\subsection{Algebraic prolongation}

Let $\tilde{G}$ be a Lie group and $G$ and $H$ two subgroups. Denote by $\tilde{\mathfrak{g}}, \mathfrak{g}$, and $\mathfrak{h}$ the respective Lie algebras seen as tangent spaces at the identity elements. We shall assume that $H$ is closed. Suppose that $\tilde{\mathfrak{g}}=\mathfrak{h} \oplus \mathfrak{g}$ and that we are given the decompositions in vector space direct sum

$$
\mathfrak{h}=V_{-h} \oplus \cdots \oplus V_{0}
$$

and

$$
\mathfrak{g}=V_{1} \oplus \cdots \oplus V_{s}
$$

in such a way that $\tilde{\mathfrak{g}}$ is graded, namely $\left[V_{i}, V_{j}\right] \subseteq V_{i+j}$, for $i, j=-h, \ldots, s$, and $\mathfrak{g}$ is stratified, i.e., $\left[V_{1}, V_{j}\right]=V_{j+1}$ for $j>0$. In other words, $\tilde{\mathfrak{g}}$ is a (finite-dimensional) prolongation of the Carnot algebra $\mathfrak{g}$.

We have a local embedding of $G$ within the quotient space $\tilde{G} / H:=\{g H: g \in G\}$ via the restriction to $G$ of the projection

$$
\pi: \tilde{G} \rightarrow \tilde{G} / H, \quad p \mapsto \pi(p):=[p]:=p H .
$$

The group $\tilde{G}$ acts on $\tilde{G} / H$ on the left:

$$
\bar{L}_{\tilde{g}}: \tilde{G} / H \rightarrow \tilde{G} / H, \quad g H \mapsto \bar{L}_{\tilde{g}}(g H):=\tilde{g} g H .
$$

We will repeatedly use the identity

$$
\bar{L}_{\tilde{g}} \circ \pi=\pi \circ L_{\tilde{g}} \text {. }
$$

On the groups $\tilde{G}$ and $G$ we consider the two left-invariant subbundles $\tilde{\Delta}$ and $\Delta$ that at the identity coincide with $\mathfrak{h}+V_{1}$ and $V_{1}$, respectively. Notice that both subbundles are bracket generating $\tilde{\mathfrak{g}}$ and $\mathfrak{g}$, respectively. Moreover, $\tilde{\Delta}$ is $\operatorname{ad}_{\mathfrak{h}}$-invariant, hence it passes to the quotient as a $\tilde{G}$-invariant subbundle $\bar{\Delta}$ on $\tilde{G} / H$. Namely, there exists a subbundle $\bar{\Delta}$ of the tangent bundle of $\tilde{G} / H$ such that

$$
\bar{\Delta}=\mathrm{d} \pi(\tilde{\Delta}) .
$$

\section{Lemma 4.3. The map}

$$
i:=\pi_{\left.\right|_{G}}:(G, \Delta) \rightarrow(\tilde{G} / H, \bar{\Delta}), \quad g \mapsto g H
$$

is a local diffeomorphism and preserves the subbundles, i.e., it is locally a contacto-morphism.

Proof. Since $\mathfrak{g}$ is a complementary subspace of $\mathfrak{h}$ in $\tilde{\mathfrak{g}}$, the differential $(\mathrm{d} i)_{e}$ is an isomorphism between $\mathfrak{g}$ and $T_{[e]} \tilde{G} / H$. Since by Equation (4.2) the map $\pi$ is $G$-equivariant, then $(\mathrm{d} i)_{g}$ is an isomorphism for any arbitrary $g \in G$. Hence, the map $i$ is a local diffeomorphism. If $X$ is a left-invariant section of $\Delta$ then

$$
(\mathrm{d} i)_{g} X_{g}=\left.\frac{\mathrm{d}}{\mathrm{d} t}\left[g \exp \left(t X_{e}\right)\right]\right|_{t=0} \in \bar{\Delta}_{[g]},
$$

since $X_{e} \in V_{1}$.

Let $\pi_{\mathfrak{g}}: \tilde{\mathfrak{g}}=V_{-h} \oplus \cdots \oplus V_{0} \oplus \mathfrak{g} \rightarrow \mathfrak{g}$ be the projection induced by the direct sum. The projections $\pi$ and $\pi_{\mathfrak{g}}$ are related by the following equation:

$$
(\mathrm{d} \pi)_{e}=\left.(\mathrm{d} \pi)_{e}\right|_{\mathfrak{g}} \pi_{\mathfrak{g}} .
$$

Indeed, if $Y \in \mathfrak{g}$, then the formula trivially holds; if $Y \in \mathfrak{h}$, then $(\mathrm{d} \pi)_{e} Y=\left.\frac{\mathrm{d}}{\mathrm{d} t} \exp (t Y) H\right|_{t=0}=\left.\frac{\mathrm{d}}{\mathrm{d} t} H\right|_{t=0}=0$.

The differential of the projection $\pi$ at an arbitrary point $\tilde{g}$ can be expressed using the projection $\pi_{\mathfrak{g}}$ via the following equation:

$$
(\mathrm{d} \pi)_{\tilde{g}}=\left(\mathrm{d}\left(\bar{L}_{\tilde{g}} \circ \pi_{\left.\right|_{G}}\right)\right)_{e} \circ \pi_{\mathfrak{g}} \circ\left(\mathrm{d} L_{\tilde{g}^{-1}}\right)_{\tilde{g}} .
$$


Indeed, first notice that $\left(\mathrm{d} \pi_{\left.\right|_{G}}\right)_{e}=(\mathrm{d} \pi)_{\left.e\right|_{\mathfrak{g}}}$, then from (4.4) and (4.2) we get

$$
\begin{aligned}
\left(\mathrm{d}\left(\bar{L}_{\tilde{g}} \circ \pi_{\mid G}\right)\right)_{e} \circ \pi_{\mathfrak{g}} \circ\left(\mathrm{d} L_{\tilde{g}^{-1}}\right)_{\tilde{g}} & =\left(\mathrm{d} \bar{L}_{\tilde{g}}\right)_{[e]} \circ(\mathrm{d} \pi)_{\left.e\right|_{\mathfrak{g}}} \circ \pi_{\mathfrak{g}} \circ\left(\mathrm{d} L_{\tilde{g}^{-1}}\right)_{\tilde{g}} \\
& =\left(\mathrm{d} \bar{L}_{\tilde{g}}\right)_{[e]} \circ(\mathrm{d} \pi)_{e} \circ\left(\mathrm{d} L_{\tilde{g}^{-1}}\right)_{\tilde{g}} \\
& =\mathrm{d}\left(\bar{L}_{\tilde{g}} \circ \pi \circ\left(L_{\tilde{g}}\right)^{-1}\right)_{\tilde{g}}=(\mathrm{d} \pi)_{\tilde{g}} .
\end{aligned}
$$

\subsection{Induced contact vector fields}

To any vector $X \in T_{e} \tilde{G} \simeq \tilde{\mathfrak{g}}$ we want to associate a contact vector field $X^{G}$ on $G$. Let $X^{R}$ be the right-invariant vector field on $\tilde{G}$ associated to $X$. We define $X^{G}$ as the (unique) vector field on $G$ with the property that

$$
\mathrm{d} \pi\left(X^{R}\right)=\mathrm{d} i\left(X^{G}\right),
$$

as vector fields on $i(G)$. In other words, we observe that there exists a (unique) vector field $\bar{X}$ on $\tilde{G} / H$ that is $\pi$-related to $X^{R}$ and $i$-related to some (unique) $X^{G}$. The flow of $X^{R}$ consists of left translations in $\tilde{G}$, hence they pass to the quotient $\tilde{G} / H$. Thus $\bar{X}$ shall be the vector field on $\tilde{G} / H$ whose flow is

$$
\Phi_{\bar{X}}^{t}(g H)=\pi(\exp (t X) g)=\exp (t X) g H=\bar{L}_{\exp (t X)}(g H) .
$$

In other words, we define $\bar{X}$ as the vector field on $\tilde{G} / H$ as

$$
\bar{X}_{[p]}:=(\mathrm{d} \pi)\left(X^{R}\right)_{p}=\left.\frac{\mathrm{d}}{\mathrm{d} t} \pi(\exp (t X) p)\right|_{t=0}, \quad \forall p \in \tilde{G} .
$$

Definition 4.7. For all $X \in \tilde{\mathfrak{g}}$ and $g \in G$, let $\left(X^{G}\right)_{g}:=\left(\mathrm{d}\left(\pi_{\left.\right|_{G}}\right)_{g}\right)^{-1}(\mathrm{~d} \pi)_{g}\left(\mathrm{~d} R_{g}\right)_{e} X$.

From (4.5), the vector field $X^{G}$ satisfies

$$
\left(X^{G}\right)_{g}=\mathrm{d}\left(L_{g_{\left.\right|_{G}}}\right)_{e} \pi_{\mathfrak{g}} \operatorname{Ad}_{g^{-1}} X, \quad \forall g \in G .
$$

We remark that if $X \in \mathfrak{g} \subset \tilde{\mathfrak{g}}$ then $X^{G}=X^{R}$, as vector fields in $G$.

Proposition 4.9. Let $X^{G}$ be the vector field defined above. Then

i) $X^{G}$ has polynomial components when read in exponential coordinates.

ii) $X^{G}$ is a contact vector field, i.e., its flow preserves $\Delta$.

Proof. Because the algebra $\tilde{\mathfrak{g}}$ is graded, we have that for every $X \in \mathfrak{g}$ the $\operatorname{map} \operatorname{ad}_{X}$ is a nilpotent transformation of $\tilde{\mathfrak{g}}$. Consequently, for all $g \in G$, the map $\operatorname{Ad}_{g}$ is a polynomial map of $\tilde{\mathfrak{g}}$. Therefore, in exponential coordinates, $X_{\left.\right|_{G}}^{R}$ is a polynomial vector field and $X^{G}$ is as well.

We next show that the vector field in (4.6) is contact, in other words, each map $\bar{L}_{p}$ preserves $\bar{\Delta}$. Any vector in $\bar{\Delta}$ is of the form $\mathrm{d} \pi\left(Y_{\tilde{g}}^{L}\right)$ with $Y_{e} \in \mathfrak{h}+V_{1}$ and $\tilde{g} \in \tilde{G}$. We want to show that $\left(\mathrm{d} \bar{L}_{p}\right)_{[\tilde{g}]}(\mathrm{d} \pi)_{\tilde{g}}\left(Y_{\tilde{g}}^{L}\right)$ is in $\bar{\Delta}$. In fact, using (4.2), we have

$$
\begin{aligned}
\left(\mathrm{d} \bar{L}_{p}\right)_{[\tilde{g}]}(\mathrm{d} \pi)_{\tilde{g}}\left(Y_{\tilde{g}}^{L}\right) & =\mathrm{d}\left(\bar{L}_{p} \circ \pi\right)_{\tilde{g}}\left(Y_{\tilde{g}}^{L}\right) \\
& =\mathrm{d}\left(\pi \circ L_{p}\right)_{\tilde{g}}\left(Y_{\tilde{g}}^{L}\right) \\
& =\mathrm{d} \pi_{p \tilde{g}}\left(Y_{p \tilde{g}}^{L}\right) \in \mathrm{d} \pi(\tilde{\Delta}) .
\end{aligned}
$$

Now that we know that $\bar{X}$ is a contact vector field of $\tilde{G} / H$, from Lemma 4.3 we deduce that the vector field $X^{G}$, which satisfies $\bar{X}=\mathrm{d} i\left(X^{G}\right)$, is a contact vector field on $G$.

For a subspace $W \subseteq \tilde{\mathfrak{g}}$ we use the notation $W^{G}:=\left\{X^{G} \in \operatorname{Vec}(G) \mid X \in W\right\}$. 
Corollary 4.10. If $\mathcal{S}$ denotes the space of global contact vector fields on $G$ that vanish at the identity, we have $\mathfrak{h}^{G} \subseteq \mathcal{S}$.

Proof. Let $X \in \mathfrak{h}$. We already proved that $X^{G}$ is a contact vector field on $G$. We only need to verify that $\left(X^{G}\right)_{e}=0$. Since $X^{G}$ is $i$-related to $\bar{X}$, it is equivalent to show that $(\bar{X})_{e}=0$, but

$$
(\bar{X})_{e}=\left.\frac{\mathrm{d}}{\mathrm{d} t} \pi(\exp (t X))\right|_{t=0}=\left.\frac{\mathrm{d}}{\mathrm{d} t} H\right|_{t=0}=0,
$$

as desired.

\subsection{A criterion for Sard's property}

For $g \in G$, denote $\mathcal{S}(g)=\{\xi(g) \mid \xi \in \mathcal{S}\}$. Also, define

$$
\mathcal{E}:=\left\{g \in G \mid\left(R_{g}\right)_{*} V_{1}+\left(L_{g}\right)_{*} V_{1}+\mathcal{S}(g)=T_{g} G\right\} .
$$

Given a horizontal curve $\gamma$ with control $u$, from Section 2.1 we know that

$$
\left(R_{\gamma(1)}\right)_{*} V_{1}+\left(L_{\gamma(1)}\right)_{*} V_{1}+\mathcal{S}(\gamma(1)) \subset \operatorname{Im}\left(\mathrm{dEnd}_{u}\right) \subset T_{\gamma(1)} G .
$$

Therefore, if the set $\mathcal{E}$ is not empty then the abnormal set is a proper subset of $G$. Moreover, observing that $\mathcal{E}$ is defined by a polynomial relation (see Proposition 4.9), we can deduce that, whenever $\mathcal{E}$ is not empty then $G$ has the (Algebraic) Sard Property.

Proposition 4.11. Let $G$ be a Carnot group and let $\tilde{G}$ and $H$ be as in the beginning of Section 4.1. Let $\mathfrak{g}$, $\tilde{\mathfrak{g}}$ and $\mathfrak{h}$ be the corresponding Lie algebras. Assume that there are $p \in \tilde{G}$ and $g \in G$ such that $p H=g H$ and

$$
\mathfrak{h}+V_{1}+\operatorname{Ad}_{p^{-1}}\left(\mathfrak{h}+V_{1}\right)=\tilde{\mathfrak{g}} .
$$

Then

$$
\left(L_{g}\right)_{*} V_{1}+\left(R_{g}\right)_{*} V_{1}+\mathfrak{h}^{G}(g)=T_{g} G .
$$

Moreover, the above formula holds for a nonempty Zariski-open set of points in $G$, and so $G$ has the Algebraic Sard Property.

Proof. Project the equation using $\pi_{\mathfrak{g}}: \mathfrak{h} \oplus \mathfrak{g} \rightarrow \mathfrak{g}$ and get

$$
V_{1}+\pi_{\mathfrak{g}} \operatorname{Ad}_{p^{-1}}\left(\mathfrak{h}+V_{1}\right)=\mathfrak{g} .
$$

Apply the differential of $\bar{L}_{p} \circ \pi_{\left.\right|_{G}}$, i.e., the map

$$
\mathrm{d}\left(\bar{L}_{p} \circ \pi_{\left.\right|_{G}}\right)_{e}: \mathfrak{g}=T_{e} G \rightarrow T_{[p]}(\tilde{G} / H)
$$

and get

$$
\mathrm{d}\left(\bar{L}_{p} \circ \pi_{\left.\right|_{G}}\right)_{e} V_{1}+\mathrm{d}\left(\bar{L}_{p} \circ \pi_{\left.\right|_{G}}\right)_{e} \pi_{\mathfrak{g}} \operatorname{Ad}_{p^{-1}}\left(\mathfrak{h}+V_{1}\right)=T_{[p]}(\tilde{G} / H) .
$$

By Equation (4.5), the left hand side is equal to

$$
\begin{aligned}
\mathrm{d} & \left(\bar{L}_{p}\right)_{[e]}(\mathrm{d} i)_{e} V_{1}+(\mathrm{d} \pi)_{p}\left(\mathrm{~d} R_{p}\right)\left(\mathfrak{h}+V_{1}\right) \\
\quad & =\mathrm{d}\left(\bar{L}_{p}\right)_{[e]}(\mathrm{d} i)_{e} V_{1}+(\mathrm{d} \pi)_{p}\left(\left(\mathfrak{h}+V_{1}\right)^{R}\right)_{p} \\
& =\mathrm{d}\left(\bar{L}_{p}\right)_{[e]}(\mathrm{d} i)_{e} V_{1}+(\mathrm{d} i)_{g}\left(\left(\mathfrak{h}+V_{1}\right)^{G}\right)_{g} \\
& =(\mathrm{d} i)_{g} \mathrm{~d}\left(L_{g}\right)_{e} V_{1}+(\mathrm{d} i)_{g}\left(\mathrm{~d} R_{g}\right)_{e} V_{1}+(\mathrm{d} i)_{g} \mathfrak{h}^{G}(g) .
\end{aligned}
$$

Now (4.12) follows because $(\mathrm{d} i)_{g}$ is an isomorphism. Since (4.12) is expressed by polynomial inequations, also the last part of the statement follows.

We give an infinitesimal version of the result above. 
Proposition 4.13. Assume that there exists $\xi \in \tilde{\mathfrak{g}}$ such that

$\mathfrak{h}+V_{1}+\operatorname{ad}_{\xi}\left(\mathfrak{h}+V_{1}\right)=\tilde{\mathfrak{g}}$.

Then there are $p \in \tilde{G}$ and $g \in G$ such that $p H=g H$ and

$$
\mathfrak{h}+V_{1}+\operatorname{Ad}_{p^{-1}}\left(\mathfrak{h}+V_{1}\right)=\tilde{\mathfrak{g}} .
$$

Proof. For all $t>0$, let $p_{t}:=\exp (t \xi)$. Take $Y_{1}, \ldots, Y_{m}$ a basis of $\mathfrak{h}+V_{1}$. Let

$$
Y_{i}^{t}:=\operatorname{Ad}_{p_{t}}\left(\frac{1}{t} Y_{i}\right)=\operatorname{ad}_{\xi}\left(Y_{i}\right)+t \sum_{k \geq 1} \frac{t^{k-2}\left(\operatorname{ad}_{\xi}\right)^{k}}{k !}\left(Y_{i}\right) .
$$

Notice that $Y_{i}^{t} \rightarrow \operatorname{ad}_{\xi}\left(Y_{i}\right)$, as $t \rightarrow 0$. Then we have

$$
\mathfrak{h}+V_{1}+\operatorname{Ad}_{p_{t}}\left(\mathfrak{h}+V_{1}\right)=\operatorname{span}\left\{Y_{1}, \ldots, Y_{m}, Y_{1}^{t}, \ldots, Y_{m}^{t}\right\} .
$$

Since

$$
\operatorname{span}\left\{Y_{1}, \ldots, Y_{m}, Y_{1}^{0}, \ldots, Y_{m}^{0}\right\}=\mathfrak{h}+V_{1}+\operatorname{ad}_{\xi}\left(\mathfrak{h}+V_{1}\right)=\tilde{\mathfrak{g}},
$$

then $Y_{1}, \ldots, Y_{m}, Y_{1}^{t}, \ldots, Y_{m}^{t}$ span the whole space $\tilde{\mathfrak{g}}$ for $t>0$ small enough. Moreover, since $p_{t} \rightarrow e \in \tilde{G}$ and hence $\left[p_{t}\right] \rightarrow[e] \in \tilde{G} / H$, for $t>0$ small enough there exists $g \in G$ such that $[g]=\left[p_{t}\right]$, because $i: G \rightarrow \tilde{G} / H$ is a local diffeomorphism at $e \in G$.

Combining Propositions 4.11 and 4.13 we obtain the following.

Corollary 4.14. Let $G$ be a Carnot group with Lie algebra $\mathfrak{g}$. Let $\tilde{\mathfrak{g}}$ and $\mathfrak{h}$ be as in the beginning of Section 4.1. Assume that there exists $\xi \in \tilde{\mathfrak{g}}$ such that

$$
\mathfrak{h}+V_{1}+\operatorname{ad}_{\xi}\left(\mathfrak{h}+V_{1}\right)=\tilde{\mathfrak{g}} .
$$

Then $G$ has the Algebraic Sard Property.

\section{Applications}

In this section we use the criteria that we established in Section 4 in order to prove items (2) to (4) of Theorem 1.2. The proof of (5) and (6) will be based on (4.1) and Corollary 4.14.

The free Lie algebra on $r$ generators is a graded Lie algebra generated freely by an $r$-dimensional vector space $V$. It thus has the form

$$
\mathfrak{f}_{r, \infty}=V \oplus V_{2} \oplus V_{3} \oplus \ldots
$$

Being free, the general linear group $G L(V)$ acts on this Lie algebra by strata-preserving automorphisms. In order to form the free $k$-step rank $r$ Lie algebra $\mathfrak{f}_{r, k}$ we simply quotient $\mathfrak{f}_{r, \infty}$ by the Lie ideal $\oplus_{s>k} V_{s}$. Thus,

$$
\mathfrak{f}_{r, k}=V \oplus V_{2} \oplus \ldots \oplus V_{k} .
$$

\subsection{Proof of (2) and (3)}

We consider the free nilpotent Lie group $F_{2,4}$ with 2 generators and step 4, and the free nilpotent Lie group $F_{3,3}$ with 3 generators and step 3 . Their Lie algebras are stratified, namely $\mathfrak{f}_{2,4}=V_{1} \oplus V_{2} \oplus V_{3} \oplus V_{4}$ and $\mathfrak{f}_{3,3}=$ $W_{1} \oplus W_{2} \oplus W_{3}$.

The Lie algebra $\mathfrak{f}_{2,4}$ is generated by two vectors, say $X_{1}, X_{2}$, in $V_{1}$, which one can complete to a basis with

$$
\begin{aligned}
& X_{21}=\left[X_{2}, X_{1}\right] \\
& X_{211}=\left[X_{21}, X_{1}\right] \quad X_{212}=\left[X_{21}, X_{2}\right] \\
& X_{2111}=\left[X_{211}, X_{1}\right] \quad X_{2112}=\left[X_{211}, X_{2}\right]=\left[X_{212}, X_{1}\right] \quad X_{2122}=\left[X_{212}, X_{2}\right] .
\end{aligned}
$$


We apply Corollary 4.14 to verify the Algebraic Sard Property for $F_{2,4}$. We take $\mathfrak{h}$ to be the space of all strata preserving derivations of $\mathfrak{f}_{2,4}$, which in this case are generated by the action of $\mathfrak{g l}(2, \mathbb{R})$ on $V_{1}$. Choose $\xi=X_{2}+$ $X_{212}+X_{2111}$. Then $\left[\xi, V_{1}\right]$ contains the vectors $X_{21}+X_{2112}$ and $X_{2122}$. Next, consider the basis $\left\{E_{i j} \mid i, j=1, \ldots, 2\right\}$ of $\mathfrak{g l}(2, \mathbb{R})$, where $E_{i j}$ denotes the matrix that has entry equal to one in the $(i, j)$-position and zero otherwise. We compute the action of the derivation defined by each one of the $E_{i j}$ 's on $\xi$. Abusing of the notation $E_{i j}$ for such derivations, an elementary calculation gives

$$
\begin{aligned}
& E_{11} \xi=X_{212}+3 X_{2111} \quad E_{12} \xi=X_{1}+X_{211} \\
& E_{22} \xi=X_{2}+2 X_{212}+X_{2111} E_{21} \xi=2 X_{2112} .
\end{aligned}
$$

Since we need to show that $V_{1}+\operatorname{ad}_{\xi} V_{1}=\mathfrak{g}$, it is enough to prove that $V_{2} \oplus V_{3} \oplus V_{4}=\left(\operatorname{ad} \xi V_{1}\right) \bmod V_{1}$, which follows from direct verification.

We consider now the case of the free nilpotent group of rank 3 and step 3 . The Lie algebra of $F_{3,3}$ is bracket generated by three vectors in $W_{1}$, say $X_{1}, X_{2}, X_{3}$, which give a basis with

$$
\begin{array}{lll}
X_{21}=\left[X_{2}, X_{1}\right] & X_{31}=\left[X_{3}, X_{1}\right] & X_{32}=\left[X_{3}, X_{2}\right] \\
X_{211}=\left[X_{21}, X_{1}\right] & X_{212}=\left[X_{21}, X_{2}\right] & X_{213}=\left[X_{21}, X_{3}\right] \\
X_{311}=\left[X_{31}, X_{1}\right] & X_{312}=\left[X_{31}, X_{2}\right] & X_{313}=\left[X_{31}, X_{3}\right] \\
X_{322}=\left[X_{32}, X_{2}\right] & X_{323}=\left[X_{32}, X_{3}\right] . &
\end{array}
$$

We have the bracket relation $\left[X_{32}, X_{1}\right]=X_{312}-X_{213}$. We apply Corollary 4.14 to verify the Algebraic Sard Property for $F_{3,3}$. We choose $\xi=X_{21}+X_{31}+X_{32}+X_{312}+X_{213}$, and we consider the action of $\mathfrak{h}$ on it. In this case $\mathfrak{h}=$ $\mathfrak{g l}(3, \mathbb{R})$. Let $E_{i j} \in \mathfrak{g l}(3, \mathbb{R})$ be the matrix that has entry equal to one in the $(i, j)$-position and zero otherwise. Then the set $\left\{E_{i j} \mid i, j=1, \ldots, 3\right\}$ is a basis of $\mathfrak{g l}(3, \mathbb{R})$. We compute the action of the elements of this basis on $\xi$. If $i \neq j$ we obtain

$$
\begin{array}{lll}
E_{12} \xi=X_{31}+X_{311} & E_{13} \xi=-X_{21}+X_{211} & E_{23} \xi=X_{21}+2 X_{212} \\
E_{21} \xi=X_{32}+X_{322} & E_{31} \xi=-X_{32}-X_{323} & E_{32} \xi=X_{31}+2 X_{313}
\end{array}
$$

whereas if $i=j$

$$
\begin{aligned}
& E_{11} \xi=X_{21}+X_{31}+X_{213}+X_{312} \\
& E_{22} \xi=X_{21}+X_{32}+X_{213}+X_{312} \\
& E_{33} \xi=X_{31}+X_{32}+X_{213}+X_{312}
\end{aligned}
$$

Next, we consider $\left[\xi, V_{1}\right]$ and notice that it contains the vectors $v=X_{212}+X_{312}+X_{322}$ and $w=X_{213}+X_{313}+X_{323}$. It is now elementary to verify that the eleven vectors $\left\{E_{i j} \xi \mid i, j=1,2,3\right\}, v$ and $w$ are linearly independent and therefore are a basis of $W_{2} \oplus W_{3}$. In conclusion, $\xi$ satisfies the hypothesis of Corollary 4.14.

Remark 5.2. In the above proof, we had to chose the element $\xi$ properly. This was done considering how $G L(3)$ acts on $F_{3,3}$. Actually, $S L(3)$ acts by graded automorphisms on $\mathfrak{f}_{3,3}$. Consequently, each layer $W_{1}, W_{2}$ and $W_{3}$ forms $S L(3)$ representations. We will see in Section 6.2 that the third layer $W_{3}$ is isomorphic to $s l(3)$ with the adjoint representation of $S L(3)$. This observation allowed us to find the element $\xi$.

\subsection{Semisimple Lie groups and associated polarized groups}

We complete here the proof of Theorem 1.2. We first recall some standard facts in the theory of semisimple Lie groups. For the details we refer the reader to [13]. To be consistent with the standard notation, only in this section we write $G$ for a noncompact semisimple Lie group and $N$ (rather than $G$ ) for the nilpotent part of a parabolic subgroup.

If $\theta$ is a Cartan involution of the semisimple Lie algebra $\mathfrak{g}$ of $G$, then the Cartan decomposition is given by the vector space direct sum

$$
\mathfrak{g}=\mathfrak{k} \oplus \mathfrak{p},
$$


where $\mathfrak{k}$ and $\mathfrak{p}$ are the eigenspaces relative to the two eigenvalues 1 and -1 of $\theta$. We fix a maximal abelian subspace $\mathfrak{a}$ of $\mathfrak{p}$, whose dimension will be denoted by $r$. Let $B$ be the Killing form on $\mathfrak{g}$; the bilinear form $\langle X, Y\rangle:=-B(X, \theta Y)$ defines a scalar product on $\mathfrak{g}$, for which the Cartan decomposition is orthogonal and by which $\mathfrak{a}$ can be identified with its dual $\mathfrak{a}^{*}$. We fix an order on the system $\Sigma \subset \mathfrak{a}^{*}$ of nonzero restricted roots of $(\mathfrak{g}, \mathfrak{a})$. Let $\mathfrak{m}=\{X \in \mathfrak{k} \mid[X, Y]=$ $0 \forall Y \in \mathfrak{a}\}$. The algebra $\mathfrak{g}$ decomposes as $\mathfrak{g}=\mathfrak{m}+\mathfrak{a}+\oplus_{\alpha \in \Sigma} \mathfrak{g}_{\alpha}$, where $\mathfrak{g}_{\alpha}$ is the root space relative to $\alpha$. We denote by $\Sigma_{+}$the subset of positive roots. The Lie algebra of $N$, denoted $\mathfrak{n}$, decomposes as the sum of (positive) restricted root spaces $\mathfrak{n}=\oplus_{\alpha \in \Sigma_{+}} \mathfrak{g}_{\alpha}$.

Proof of (4). Denote by $\Pi_{+}$the subset of positive simple roots. The space $V=\oplus_{\delta \in \Pi_{+}} \mathfrak{g}_{\delta}$ provides a stratification of $\mathfrak{n}$, so that $(N, V)$ is a Carnot group. We prove that $(N, V)$ has the Algebraic Sard Property. Let $w$ be a representative in $G$ of the longest element in the analytic Weyl group. From [13, Theorem 6.5] we have $\operatorname{Ad} w^{-1} \overline{\mathfrak{n}}=\mathfrak{n}$, where $\overline{\mathfrak{n}}=\oplus_{\alpha \in-\Sigma_{+}} \mathfrak{g}_{\alpha}$. The Bruhat decomposition of $G$ shows that $N$ may be identified with the dense open subset $N \bar{P}$ of the homogeneous space $G / \bar{P}$, where $\bar{P}$ denotes the minimal parabolic subgroup of $G$ containing $\bar{N}$. Here we wrote $\bar{N}$ for the connected nilpotent Lie group whose Lie algebra is $\overline{\mathfrak{n}}$. Now we apply Proposition 4.11 to $\mathfrak{h}=\mathfrak{m}+\mathfrak{a}+\overline{\mathfrak{n}}$. From our discussion it follows that $\mathfrak{h}+\operatorname{Ad} w^{-1} \mathfrak{h}=\mathfrak{g}$. This equality holds true in a small neighborhood of $w$, so by density we can find $p$ in $G$ such that $[p]=[n]$ for some $n \in N$ and for which $\mathfrak{h}+\operatorname{Ad}^{-1} \mathfrak{h}=\mathfrak{g}$. Then by Proposition 4.11 we conclude that the desired Sard's property for $N$ follows.

Proof of (5). From the properties of the Cartan decomposition it follows that $[\mathfrak{p}, \mathfrak{p}]=\mathfrak{k}$. Then $(G, \mathfrak{p})$ is a polarized group. We restrict to the case where $\mathfrak{g}$ is the split real form of a complex semisimple Lie algebra. In order to show that $(G, \mathfrak{p})$ has the Analytic Sard Property, we show that there is $\xi \in \mathfrak{a}$ such that $\operatorname{ad}_{\xi} \mathfrak{p}=\mathfrak{k}$. If this holds, then by a similar argument of that in the proof of Proposition 4.13 we also have $\mathfrak{p}+\operatorname{Ad}_{g} \mathfrak{p}=\mathfrak{g}$ for some $g \in G$, from which we deduce the Analytic Sard Property. Let then $\xi$ be a regular element in $\mathfrak{a}$. This implies in particular that $\xi$ is such that $\alpha(\xi) \neq 0$ for every root $\alpha$. Next, observe that for every $\alpha \in \Sigma$ and $X \in \mathfrak{g}_{\alpha}$, we may write

$$
X=\frac{1}{2}(X-\theta X)+\frac{1}{2}(X+\theta X)
$$

where $X-\theta X \in \mathfrak{p}$ and $X+\theta X \in \mathfrak{k}$. We obtain

$$
[\xi, X-\theta X]=\alpha(\xi) X-\theta[\theta \xi, X]=\alpha(\xi)(X+\theta X) .
$$

The assumption that $\mathfrak{g}$ is split implies in particular that $\mathfrak{k}$ is generated by vectors of the form $X+\theta X$, with $X$ a nonzero vector in a root space. Since $\xi$ is regular, it follows that $\operatorname{ad}_{\xi} \mathfrak{p}=\mathfrak{k}$, which concludes the proof.

We observe that if $\mathfrak{g}$ is not split, then we do not find a vector $\xi$ such that $\mathfrak{p}+\operatorname{ad}_{\xi} \mathfrak{p}=\mathfrak{g}$ and so the same proof does not work. This can be shown, for example, by an explicit calculation on $\mathfrak{g}=\mathfrak{s u}(1,2)$.

Proof of (6). We observe that $\left(G, \oplus_{\alpha \in \Sigma} \mathfrak{g}_{\alpha}\right)$ is a polarized group. Also in this case we assume that $\mathfrak{g}$ is split. This implies that every root space $\mathfrak{g}_{\alpha}, \alpha \in \Sigma$, is one dimensional, and that $\mathfrak{m}=\{0\}$. We recall that the Killing form $B$ identifies $\mathfrak{a}$ with $\mathfrak{a}^{*}$. Let $H_{\alpha} \in \mathfrak{a}$ be such that $\alpha(H)=B\left(H_{\alpha}, H\right)$ for every $H \in \mathfrak{a}$. Recall that $\left[X_{\alpha}, \theta X_{\alpha}\right]=B\left(X_{\alpha}, \theta X_{\alpha}\right) H_{\alpha}$ and $B\left(X_{\alpha}, \theta X_{\alpha}\right)<0$. Let $\delta_{1}, \ldots, \delta_{r}$ be a basis of simple roots, and let $X_{\delta_{i}}$ be a basis of $\mathfrak{g}_{\delta_{i}}$ for every $i=1, \ldots, r$. The set of vectors $\left\{H_{\delta_{1}}, \ldots, H_{\delta_{r}}\right\}$ is a basis of $\mathfrak{a}$. Then the vector

$$
\xi=X_{\delta_{1}}+\cdots+X_{\delta_{r}}
$$

satisfies $\left[\xi, \oplus_{\alpha \in \Sigma} \mathfrak{g}_{\alpha}\right] \supset \mathfrak{a}$, whence $\oplus_{\alpha \in \Sigma} \mathfrak{g}_{\alpha}+\left[\xi, \oplus_{\alpha \in \Sigma} \mathfrak{g}_{\alpha}\right]=\mathfrak{g}$. Arguing as in the Proof of (5), we conclude that $\left(G, \oplus_{\alpha \in \Sigma} \mathfrak{g}_{\alpha}\right)$ has the Analytic Sard Property.

\subsection{Sard Property for some semidirect products}

In this section we construct polarized groups that are not nilpotent and yet have the Algebraic Sard Property. These examples are constructed as semidirect products.

Let $\psi: H \rightarrow \operatorname{Aut}(G)$ be an action of a Lie group $H$ on a Lie group $G$, i.e., $\psi$ is a continuous homomorphism from $H$ to the group of automorphisms of $G$. Write $\psi_{h}$ for $\psi(h)$, for $h \in H$. The semidirect product $G \rtimes_{\psi} H$ has product 


$$
\left(g_{1}, h_{1}\right) \cdot\left(g_{2}, h_{2}\right)=\left(g_{1} \psi_{h_{1}}\left(g_{2}\right), h_{1} h_{2}\right)
$$

Let $V \subseteq \mathfrak{g}$ be a polarization for $G$. Assume that

$$
\left(\psi_{h}\right)_{*}(V)=V, \quad \text { for all } h \in H .
$$

We consider the group $G \rtimes_{\psi} H$ endowed with the polarization $V \oplus \mathfrak{h}$, where $\mathfrak{h}$ is the Lie algebra of $H$.

Proposition 5.5. Assume that $H \stackrel{\psi}{\curvearrowright} G$ is an action satisfying (5.4). If $(G, V)$ has the Algebraic Sard Property, so does $\left(G \rtimes_{\psi} H, V \oplus \mathfrak{h}\right)$.

Proof. We show that $\operatorname{Abn}_{G \rtimes_{\psi} H}(e)$ is contained in $\operatorname{Abn}_{G}(e) \cdot H$. It is a consequence of (5.4) that a curve $\gamma(t)=$ $(g(t), h(t))$ in $\tilde{G}:=G \rtimes_{\psi} H$ is horizontal with respect to $V+\mathfrak{h}$ if and only if $g(t)$ is horizontal in $G$ and $h(t)$ is horizontal in $H$.

Hence, if $g(1) \notin \operatorname{Abn}_{G}(e)$, i.e., $g$ is not abnormal, from (2.4), we have

$$
\begin{aligned}
\left(\mathrm{dR}_{\gamma(1)}\right)_{e}^{-1} \operatorname{Im}\left(\operatorname{dEnd}_{u_{\gamma}}\right) & =\operatorname{span}\left\{\operatorname{Ad}_{\gamma(t)}(V \oplus \mathfrak{h}) \mid t \in[0,1]\right\} \\
& \supseteq V+\mathfrak{h}+\operatorname{span}\left\{\operatorname{Ad}_{\gamma(t)} V \mid t \in(0,1]\right\} \\
& =V+\mathfrak{h}+\operatorname{span}\left\{\operatorname{Ad}_{(g(t), 0)} \operatorname{Ad}_{(0, h(t))} V \mid t \in(0,1]\right\} \\
& =V+\mathfrak{h}+\operatorname{span}\left\{\operatorname{Ad}_{(g(t), 0)} V \mid t \in(0,1]\right\} \\
& =\mathfrak{g}+\mathfrak{h},
\end{aligned}
$$

where we used first that $\left(g, e_{H}\right) \cdot\left(e_{G}, h\right)=(g, h)$ and $\operatorname{Ad}_{\left(e_{G}, h\right)}(v, 0)=\left(\left(\mathrm{d} \psi_{h}\right)_{e} v, 0\right)$; then we used the assumption (5.4) and the fact $\operatorname{Ad}_{\left(g, e_{H}\right)}(v, 0)=\left(\operatorname{Ad}_{g} v, 0\right)$.

Remark 5.6. If $(G, V)$ is a free nilpotent Lie group for which the Algebraic Sard Property holds, we may take $H$ to be any subgroup of $G L(n, V)$ and apply the proposition above to $G \rtimes H$. If $(N, V)$ is a Carnot group as in the first part of Section 5.2, then $\mathfrak{h}$ may be chosen to be any subalgebra of $\mathfrak{m} \oplus \mathfrak{a}$. In particular, the Algebraic Sard Property holds for exponential growth Lie groups $N A$ if $N$ has step 2.

\section{Step-3 Carnot groups}

Our first goal in this section is to prove Theorem 1.5 concerning the Sard Property for length minimizers in Carnot groups of step 3. A secondary goal is to motivate the claim made in Example 3.7 that the typical abnormal curve in $F_{3,3}$, the free 3-step rank-3 Carnot group, does not lie in any proper subgroup. To this purpose we illustrate the beautiful structure of the abnormal equations in this case.

\subsection{Sard Property for abnormal length minimizers}

In [19] Tan and Yang proved that in sub-Riemannian step-3 Carnot groups all length minimizing curves are smooth. They also claim that in this setting all abnormal length minimizing curves are normal. Hence, Theorem 1.5 would immediately follow from Lemma 2.31. Being unable to follow some of the proofs in [19], we prefer to provide here an independent proof of Theorem 1.5, which relies on the weaker claim that every length-minimizing curve is normal in some Carnot subgroup.

Proof of Theorem 1.5. By Lemma 2.31, it is enough to estimate the set $\operatorname{Abn}_{s t r}^{l m}(e)$ of points connected to $e$ by strictly abnormal length minimizers. Let $\gamma$ be such a curve starting from the origin $e$ of a Carnot group $G$ of step 3. Since $\gamma$ is not normal, then it satisfies the Goh condition; in particular, $\gamma$ is contained in the algebraic variety

$$
W^{\lambda}=\left\{g \in G: \lambda\left(\operatorname{Ad}_{g} V_{2}\right)=0\right\}
$$

for some $\lambda \in \mathfrak{g}^{*} \backslash\{0\}$. We now use Remark 2.35, Remark 2.25, and the fact that $G$ is of step-3 to deduce that $\lambda \in$ $V_{3}^{*} \backslash\{0\}$ and that $W^{\lambda}$ is a proper subgroup of $G$. Hence also the accessible set $H^{\lambda}$ in $W^{\lambda}$ is a proper Carnot subgroup of $G$. 
Since $\gamma$ is still length minimizing in $H^{\lambda}$, either $\gamma$ is normal in $H^{\lambda}$, and we stop, or, being length minimizing, it is strictly abnormal (i.e., abnormal but not normal) in $H^{\lambda}$, and we iterate. Eventually, we obtain that $\gamma$ is normal within a Carnot subgroup. We remark that in this subgroup $\gamma$ may be abnormal or not abnormal. We do not need divide the two cases. We decompose

$$
\operatorname{Abn}_{s t r}^{l m}(e) \subseteq \bigcup_{G^{\prime}<G} \operatorname{Abn}_{G^{\prime}}^{n o r}(e),
$$

where $\operatorname{Abn}_{G^{\prime}}^{n o r}(e)$ is the union of all curves starting from $e$ that are contained in $G^{\prime}$, are normal in $G^{\prime}$, and are abnormal within $G$.

The idea is now to adapt the argument of Lemma 2.31 for the union of the sets $\operatorname{Abn}_{G^{\prime}}^{\text {nor }}(e)$. Carnot subgroups of $G$ are parametrized by the Grassmannian of linear subspaces of $V_{1}$. The dimension of the subgroup is a semi-algebraic function on the Grassmannian. On each of its level sets $Y_{m}$, all relevant data (e.g., coefficients of the Hamiltonian equation satisfied by normal length minimizing curves) are real analytic. The dual Lie algebras $\mathfrak{g}^{*}$ form an analytic vector bundle over $Y_{m}$. Denote by $\tau_{m}$ the total space of this bundle. It is a semi-analytic subset of $T_{e}^{*} G$. The time 1 solutions of the Hamiltonian equations with initial data in $\tau_{m}$ give rise to real analytic maps $\widetilde{\operatorname{Exp}}_{m}: \tau_{m} \rightarrow L^{2}([0,1], V)$. Each subgroup has its own geodesic exponential map, giving rise to an analytic map $\operatorname{Exp}_{m}: \tau_{m} \rightarrow G$. Again,

$$
\operatorname{Exp}_{m}=\text { End } \circ \widetilde{\operatorname{Exp}}_{m} \text {. }
$$

Every point in $\bigcup_{G^{\prime}<G} \operatorname{Abn}_{G^{\prime}}^{\text {nor }}(e)$ is a value of some $\operatorname{Exp}_{m}$ where the differential of End is not onto. Therefore, it is a singular value of $\operatorname{Exp}_{m}$. This constitutes a measure zero sub-analytic subset of $G$.

Remark 6.1. In the free 3 -step Carnot group, we are not able to bound the codimension of $\mathrm{Abn}^{l m}(e)$ away from 1 . However, the codimension of $\operatorname{Abn}_{s t r}^{l m}(e)$ is at least 3. Actually, in the free 3-step rank-r group $\mathbb{F}_{r, 3}$ this codimension is greater or equal than $r^{2}-r+1$. The calculation is similar to the one in Section 3.5. Indeed, by the Witt Formula (see [9, pp. 140-142]) the dimension of $\mathbb{F}_{r, 3}$ is

$$
\operatorname{dim} \mathbb{F}_{r, 3}=r+\frac{r(r-1)}{2}+\frac{r^{3}-r}{3} .
$$

In the proof of Theorem 1.5, we showed that each abnormal geodesic from the origin is in a subgroup, which therefore has codimension bounded by $\operatorname{dim} \mathbb{F}_{r-1,3}$, computable via the Witt Formula (6.2). The collection of all the subgroups of rank $r-1$ can be parametrized via the Grassmannian $\operatorname{Gr}(r, r-1)$, which has dimension $r-1$. Therefore, we compute

$$
\operatorname{dim} \mathbb{F}_{r, 3}-\operatorname{dim} \mathbb{F}_{r-1,3}-\operatorname{dim} G r(r, r-1)=r^{2}-r+1 .
$$

Notice that $r^{2}-r+1$ equals 3 if $r=2$, and is strictly greater than 7 if $r \geq 3$.

\subsection{Investigations in the rank-3 case}

As said in Section 5, the group $G L(V)$ acts on each strata $V_{j}$ of the free algebra $\mathfrak{f}_{r, \infty}$. So each summand $V_{j}$ breaks up into $G L(V)$ irreducibles. Also, the $k$-step rank $r$ Lie algebra decomposes as a representation space

$$
\mathfrak{f}_{r, k}=V \oplus V_{2} \oplus \ldots \oplus V_{k} .
$$

The first summand $V$ is the 'birthday representation' of $G L(V)$. The second summand is well-known as a $G L(V)$ representation, and in any case is easy to guess:

$$
V_{2}=\Lambda^{2} V
$$

with the Lie bracket $V \times V \rightarrow \Lambda^{2} V$ being $[v, w]=v \wedge w$. The third summand is less well-known and will be treated momentarily. First a few more generalities. Any algebra becomes a Lie algebra when we define the Lie bracket between two elements to be their commutator. So the full tensor algebra $\mathfrak{T}(V)=V \oplus V^{\otimes 2} \oplus V^{\otimes 3} \oplus \cdots$ inherits a Lie algebra structure. Under this bracket we have $[v, w]=v \otimes w-w \otimes v=v \wedge w$ for $v, w \in V$. The free Lie algebra 
over $V$ is the Lie subalgebra that is Lie-generated by $V$ within the full tensor algebra $\mathfrak{T}(V)$. In particular,

$$
V_{r} \subset V^{\otimes r} .
$$

Both the symmetric group $S_{r}$ on $r$ letters, and the general linear group $G L(V)$ acts on $V^{\otimes r}$. By Schur-Weyl duality, see [10, Exercise 6.30, p. 87], under the joint action of $G L(V) \times S_{r}$ the space $V^{\otimes r}$ breaks up completely into irreducibles and this representation is "multiplicity free": each irreducible occurs at most once. The irreducibles themselves are written in the form $S_{\lambda}(V) \otimes \operatorname{Specht}(\lambda)$. Here $\lambda$ is a partition of $r$ and is represented by a Young Tableaux with blank boxes. Then $S_{\lambda}(V)$ is the irreducible representation of $G L(V)$ corresponding to $\lambda$, whereas $\operatorname{Specht}(\lambda)$ is the irreducible representation of $S_{r}$ corresponding to this $\lambda$. If we are only interested in decomposing $V^{\otimes r}$ into $G L(V)$-irreducibles, what this means is that each irreducible $S_{\lambda}(V)$ occurs $\operatorname{dim}(\operatorname{Specht}(\lambda))$ times. For example, the representation $S^{r}(V)$ of symmetric powers of $V$ corresponds to the partition $r=1+1+1+\cdots+1$. The representation $\Lambda^{r}(V)$ corresponds to the partition $r=r$.

To the case at hand, $V_{3} \subset V^{\otimes 3}$ corresponds to the partition $3=2+1$. This representation is dealt with in fine detail in [10, pp. 75-76]. We summarize the results within our context. The bracket map $V \otimes \Lambda^{2} V \rightarrow V_{3}$ which sends $v \otimes \omega \rightarrow[v, \omega]=v \otimes \omega-\omega \otimes v$ is onto, but as soon as $\operatorname{dim}(V)>2$ it is not injective due to the Jacobi identity. We want to describe the image $V_{3}$ of the bracket map. There is a canonical inclusion $i: V \otimes \Lambda^{2} V \rightarrow V^{\otimes 3}$, namely the identity $v \otimes \omega \mapsto v \otimes \omega$, whose image contains $V_{3}$. To cut $V \otimes \Lambda^{2} V \subset V^{\otimes 3}$ down to $V_{3}$ we must add linear conditions which encode the Jacobi identity. Consider the canonical projection map $\beta: V^{\otimes 3} \rightarrow \Lambda^{3} V$ which sends $v_{1} \otimes v_{2} \otimes v_{3}$ to $v_{1} \wedge v_{2} \wedge v_{3}$. Then the Jacobi identity is $\beta=0$, so that $V_{3}=\operatorname{im}(i) \cap \operatorname{ker}(\beta)$.

Let us now go to the specific case of $\operatorname{dim}(V)=3$. Here $\operatorname{dim}\left(V \otimes \Lambda^{2} V\right)=3 \times 3=9$, whereas $\operatorname{dim}\left(V_{3}\right)=8$. In this case the Jacobi identity is 'one-dimensional'. We show how to identify $V_{3}$ with $\mathfrak{s l}(3)$ by fixing a volume form on $V$. Write coordinates $x, y, z=x_{1}, x_{2}, x_{3}$ on $V$ and take as the resulting volume form $\mu=d x_{1} \wedge d x_{2} \wedge d x_{3}$. The choice of form both singles out $S L(3) \subset G L(3)=G L(V)$ and yields a canonical identification $\Lambda^{2} V \cong V^{*}$ by sending $v \wedge w$ to the one-form $\mu(v, w, \cdot)$. Thus $V \otimes \Lambda^{2} V \cong V \otimes V^{*}=\mathfrak{g l}(V)$ as an $S L(3)$ representation space, with $\operatorname{SL}(3)=\operatorname{SL}(V)$ acting by conjugation on $\mathfrak{g l}(V)$. For example, $\partial_{j} \otimes\left(\partial_{1} \wedge \partial_{2}\right)$ is sent to the element $\partial_{j} \otimes d x_{3}$ under this identification. One verifies that the kernel of $\beta$ is equal to the span of the identity element $I=\partial_{1} \otimes d x_{1}+\partial_{2} \otimes d x_{2}+\partial_{3} \otimes d x_{3}$ under this identification. Thus $V_{3} \cong \mathfrak{g l}(V) / \mathbb{R} I$. Next, observe that as an $S L(V)$ (or $G L(V)$ ) representation space we have: $V \otimes V^{*}=\mathfrak{s l}(V) \oplus \mathbb{R} I$ where $\mathfrak{s l}(V)$ consists of those matrices with trace zero. Thus $V_{3}=\mathfrak{g l}(V) / \mathbb{R} I=\mathfrak{s l}(V)$, as $S L(V)$ representation spaces. Notice that as $G L(V)$ representation spaces this equality does not hold since the element $\lambda I \in G L(V)$ acts on $V_{3}$ by $\lambda^{3} I$, while under conjugation the same element acts on $\mathfrak{s l}(V)$ as the identity. An investigation of what $\operatorname{ad}_{\xi}$ looks like in relation to this $S L(3)$-equivariant decomposition led to the specific element $\xi$ defined at the end of Section 5.1.

To get to the equations describing abnormality for $F_{3,3}$, we write its Lie algebra as

$$
\mathfrak{f}_{3,3}=V_{1} \oplus V_{2} \oplus V_{3}=\mathbb{R}^{3} \oplus \mathbb{R}^{3 *} \oplus \mathfrak{s l}(3)
$$

and so an element of the dual Lie algebra can be written out as

$$
\lambda=\left(\lambda_{1}, \lambda_{2}, \lambda_{3}\right) \in \mathfrak{f}_{3,3}^{*}=V_{1}^{*} \oplus V_{2}^{*} \oplus V_{3}^{*}=\mathbb{R}^{3 *} \oplus \mathbb{R}^{3} \oplus \mathfrak{s l}(3)^{*} .
$$

For this covector to lie along an abnormal extremal it must be $\lambda_{1}=0$.

We partition the abnormal extremals into two classes: those for which $\lambda_{2} \neq 0$, which we call regular abnormal extremals following Liu-Sussmann, and those for which $\lambda_{2}=0$. The Hamiltonian

$$
H=P_{1} P_{23}+P_{2} P_{31}+P_{3} P_{12}
$$

generates all the regular abnormal extremals. Here

$$
\begin{aligned}
& \lambda_{1}=\left(P_{1}, P_{2}, P_{3}\right) \\
& \lambda_{2}=\left(P_{23}, P_{31}, P_{12}\right)
\end{aligned}
$$

and

$$
P_{i}=P_{X_{i}} \quad P_{i j}=P_{X_{i j}}=-P_{j i}
$$


where we are following the notation of (2.19) and (5.1). When we say that $H$ "generates" the regular abnormal extremals we mean two things: (A) the Hamiltonian flow of $H$ preserves the locus $\lambda_{1}=0$, i.e., the locus $\Delta^{\perp}=$ $\left\{P_{1}=P_{2}=P_{3}=0\right\}$ and (B) on the locus $\lambda_{1}=0, \lambda_{2} \neq 0$, a unique - up to reparameterization - abnormal extremal passes through every point, with the extremal through $\left(0, \lambda_{2}, \lambda_{3}\right)$ being the solution to Hamilton's equations for this Hamiltonian $H$ with initial conditions $\lambda$.

We follow a Hamiltonian trick that Igor Zelenko kindly showed us for both finding $H$ and for validating claims (A) and (B). Start with the Maximum Principle characterization of abnormal extremals discussed in Section 2.4. According to this principle, an abnormal with control $u(t)$ is a solution to Hamilton's equations having the time dependent Hamiltonian $H_{u}=u_{1} P_{1}+u_{2} P_{2}+u_{3} P_{3}$ and lying in the common level set $P_{1}=0, P_{2}=0, P_{3}=0$. From Hamilton's equations we find that

$$
\begin{aligned}
& \dot{P}_{1}=\left\{P_{1}, H_{u}\right\}=-u_{2} P_{12}-u_{3} P_{13} \\
& \dot{P}_{2}=\left\{P_{2}, H_{u}\right\}=-u_{1} P_{21}-u_{3} P_{23} \\
& \dot{P}_{3}=\left\{P_{3}, H_{u}\right\}=-u_{1} P_{31}-u_{2} P_{32}
\end{aligned}
$$

But we must have that $\dot{P}_{i}=0$. Consequently $\left(u_{1}, u_{2}, u_{3}\right)$ must lie in the kernel of the skew-symmetric matrix whose entries are $P_{i j}$. As long as this matrix is not identically zero, its kernel is one-dimensional and is spanned by $\left(P_{23}, P_{31}, P_{12}\right)$. It follows that:

$$
\left(u_{1}, u_{2}, u_{3}\right)=f\left(P_{23}, P_{31}, P_{12}\right), \quad f \neq 0 .
$$

Since the parameterization of the abnormal is immaterial, we may take $f=1$. Plugging our expression for $u$ back in to $H_{u}$ yields the form of $H$ above.

We can write down the ODEs governing the regular abnormal extremals, using this $H$. We have just seen that

$$
u=\lambda_{2}=\left(P_{23}, P_{31}, P_{12}\right)
$$

describes the controls, i.e., the moving element of $V$. This control evolves according to

$$
\dot{u}=A u
$$

where $A$ is a constant matrix in $S L(3)$. These are to be supplemented by the understanding of what the resulting abnormal extremal is

$$
\lambda_{1}=0, \quad \lambda_{2}=u, \quad \lambda_{3}=A .
$$

We want to establish Hamilton's equations, using this $H$. For doing so, we compute $\dot{P}_{i j}=\left\{P_{i j}, H\right\}$ and $\dot{P}_{i j k}=$ $\left\{P_{i j k}, H\right\}=0$ where $P_{i j k}=P_{X_{i j k}}$. The first equation results in a bilinear pairing between $P_{i j}$ and $P_{i j k}$ which, when the $P_{i j k}$ are properly interpreted as an element $A \in S L(3)$, is matrix multiplication.

\subsection{Computation of abnormals not lying in any subgroup}

Take a diagonalizable $A$ with distinct nonzero eigenvalues $a, b, c, a+b+c=0$. For simplicity, let it be $\operatorname{diag}(a, b, c)$ relative to our choice of coordinates for $V$. Then $u$ evolves according to $u(t)=\left(A e^{a t}, B e^{b t}, C e^{c t}\right)$. We may suppose that none of $A, B, C$ are zero by assuming that no components of $\lambda_{2}=u(0)$ are zero. The corresponding curve in $G$ passing through $e=0$, projected onto the first level is the curve $x_{1}=\frac{1}{a}\left(A\left(e^{a t}-1\right), x_{2}=\frac{1}{b}\left(B\left(e^{b t}-1\right), x_{3}=\right.\right.$ $\frac{1}{c}\left(C\left(e^{c t}-1\right)\right.$. Since the functions $1, e^{a t}, e^{b t}, e^{c t}$ are linearly independent, the curve projected to the first level cannot lie in any proper subspace of $V$, which in turn implies that the entire abnormal curve cannot lie in any proper subgroup of $G$.

Alternatively, one can directly use Corollary 2.14. In fact, with the notation of Section 5, one can take $\lambda=e_{21}^{*}-$ $e_{31}^{*}+e_{32}^{*}-c e_{213}^{*}+b e_{312}^{*}$ to prove that the curve with control $u(t)=\left(e^{(-b-c) t}, e^{b t}, e^{c t}\right)$ is abnormal.

The characteristic viewpoint. We put forth one further perspective on abnormal extremals which makes the computation just done more transparent. Take any polarized manifold $(Q, \Delta)$. Take the annihilator bundle of $\Delta$, denoted $\Delta^{\perp} \subset T^{*} Q$. Restrict the canonical symplectic form $\omega$ of $T^{*} Q$ to $\Delta^{\perp}$. Call this restriction $\omega_{\Delta}$. Then the abnormal 
extremals are precisely the (absolutely continuous) characteristics for $\omega_{\Delta}$, that is the curves in $\Delta^{\perp}$ whose tangents are a.e. in $\operatorname{Ker}\left(\omega_{\Delta}\right)$. Let $\pi: \Delta^{\perp} \rightarrow Q$ be the canonical projection. Then a linear algebra computation shows that $d \pi_{(q, \lambda)}$ projects $\operatorname{Ker}\left(\omega_{\Delta}\right)(q, \lambda)$ linearly isomorphically onto $\operatorname{Ker}\left(w_{q}(\lambda)\right) \subset \Delta_{q}$ where $\lambda \in \Delta_{q}^{\perp} \mapsto w_{q}(\lambda) \in \Lambda^{2} \Delta_{q}^{*}$ is the operator called the "dual curvature" in [17]. In the case of a polarized group $(Q, \Delta)=(G, V)$ we have that $w_{q}(\lambda)$ is the two-form of Equation (2.15) for $\lambda=\eta \in V^{\perp}$.

In our situation $V$ has dimension 3 so that $w(\lambda)$ has either rank 2 or 0 and thus its kernel has dimension 1 or 3. The kernel has dimension 1 exactly when $\lambda_{2} \neq 0$, and rank 3 exactly when $\lambda_{2}=0$. Along the points where $\lambda_{2} \neq 0$ the kernel of $\omega_{\Delta}$ is a line field, and the Hamiltonian vector field $X_{H}$ for $H$ above rectifies this line field. Note that $X_{H}$ vanishes exactly along the variety $\lambda_{2}=0$.

\section{Open problems}

Is $\operatorname{Abn}(e)$, the set of endpoints of abnormal extremals leaving the identity, a closed analytic variety in $G$ when $G$ is a simply connected polarized Lie group? In all examples computed, the answer is 'yes'. However, even the following more basic questions are still open. Is $\operatorname{Abn}(e)$ closed? Can $\operatorname{Abn}(e)$ be the entire group $G$ ?

Concerning the importance of the adjective "simply connected" above, consider the torus. Any integrable distribution $V$ whose corank is 1 or greater on any space $G$ has its $\operatorname{Abn}(e)$ the leaf through $e$. Consequently an irrationally oriented polarization $V$ on the torus has for its $\operatorname{Abn}(e)$ a set that is neither closed nor analytic. We also ask whether statements 5 and 6 of Theorem 1.2 can be upgraded to algebraic. Can one unify (6) and (7) having the result for all semisimple groups? If $G$ and $H$ are polarized Lie groups having the Sard Property, does any semidirect product $G \rtimes H$ have the Sard Property? Finally, in the particular case of rank 2 Carnot groups, what is the minimal codimension of $\operatorname{Abn}(e)$ ?

\section{Conflict of interest statement}

The authors have declared no conflicts of interest.

\section{Acknowledgements}

Most of the work in this paper was developed while the authors were guests of the program Geometry, Analysis and Dynamics on Sub-Riemannian Manifolds at the Institut Henri Poincaré in the Fall 2014. The authors are very grateful to the program organizers A. Agrachev, D. Barilari, U. Boscain, Y. Chitour, F. Jean, L. Rifford, and M. Sigalotti, as well to IHP for its support.

\section{References}

[1] Andrei Agrachev, Davide Barilari, Ugo Boscain, Introduction to Riemannian and sub-Riemannian geometry, Manuscript, available at http://webusers.imj-prg.fr/ davide.barilari, 2015.

[2] Andrei Agrachev, Alessandro Gentile, Antonio Lerario, Geodesics and admissible-path spaces in Carnot groups, arXiv:1311.6727, 2013.

[3] Andrei Agrachev, Any sub-Riemannian metric has points of smoothness, Dokl. Akad. Nauk 424 (3) (2009) 295-298.

[4] Andrei Agrachev, Some open problems, arXiv:1304.2590, 2013.

[5] Vladimir I. Arnol'd, Matrices depending on parameters, Usp. Mat. Nauk 26 (2(158)) (1971) 101-114.

[6] Andrei Agrachev, Andrei Sarychev, Abnormal sub-Riemannian geodesics: Morse index and rigidity, Ann. Inst. Henri Poincaré, Anal. Non Linéaire 13 (6) (1996) 635-690.

[7] Andrei Agrachev, Yuri Sachkov, Control Theory from the Geometric Viewpoint. Control Theory and Optimization, II, Encyclopaedia Math. Sci., vol. 87, Springer-Verlag, Berlin, 2004.

[8] Jacek Bochnak, Michel Coste, Marie-Francoise Roy, Real Algebraic Geometry, Ergeb. Math. Ihrer Grenzgeb. (3) (Results Math. Relat. Areas (3)), vol. 36, Springer-Verlag, Berlin, 1998; Translated from the 1987 French original; Revised by the authors.

[9] Nicolas Bourbaki, Lie Groups and Lie Algebras, Chapters 1-3, Elem. Math. (Berlin), Springer-Verlag, Berlin, 1998; Translated from the French; Reprint of the 1989 English translation, MR1728312 (2001g:17006).

[10] William Fulton, Joe Harris, Representation Theory: A First Course, Grad. Texts Math.: Read. Math., vol. 129, Springer-Verlag, New York, 1991.

[11] Christophe Golé, Ron Karidi, A note on Carnot geodesics in nilpotent Lie groups, J. Dyn. Control Syst. 1 (4) (1995) 535-549.

[12] Mikhail Gromov, Carnot-Carathéodory Spaces Seen from within, Sub-Riemannian Geometry, Prog. Math., vol. 144, Birkhäuser, Basel, 1996, pp. 79-323. 
[13] Anthony W. Knapp, Lie Groups Beyond an Introduction, second ed., Prog. Math., vol. 140, Birkhäuser Boston Inc., Boston, MA, 2002.

[14] Enrico Le Donne, Gian Paolo Leonardi, Roberto Monti, Davide Vittone, Extremal curves in nilpotent Lie groups, Geom. Funct. Anal. 23 (4) (2013) 1371-1401.

[15] Enrico Le Donne, Gian Paolo Leonardi, Roberto Monti, Davide Vittone, Extremal polynomials in stratified groups, Preprint, 2014, submitted for publication, available at http://arxiv.org/abs/1307.5235.

[16] Richard Montgomery, Singular extremals on Lie groups, Math. Control Signals Syst. 7 (3) (1994) 217-234.

[17] Richard Montgomery, A Tour of Subriemannian Geometries, Their Geodesics and Applications, Math. Surv. Monogr., vol. 91, American Mathematical Society, Providence, RI, 2002.

[18] Ludovic Rifford, Emmanuel Trélat, Morse-Sard type results in sub-Riemannian geometry, Math. Ann. 332 (1) (2005) $145-159$.

[19] Kanghai Tan, Xiaoping Yang, Subriemannian geodesics of Carnot groups of step 3, ESAIM Control Optim. Calc. Var. 19 (1) (2013) $274-287$. 